J. Korean Math. Soc. 48 (2011), No. 6, pp. 1285-1325

http://dx.doi.org/10.4134/JKMS.2011.48.6.1285

\title{
THE ISOPERIMETRIC PROBLEM ON EUCLIDEAN, SPHERICAL, AND HYPERBOLIC SURFACES
}

\author{
Matthew D. Simonson
}

\begin{abstract}
We solve the isoperimetric problem, the least-perimeter way to enclose a given area, on various Euclidean, spherical, and hyperbolic surfaces, sometimes with cusps or free boundary. On hyperbolic genustwo surfaces, Adams and Morgan characterized the four possible types of isoperimetric regions. We prove that all four types actually occur and that on every hyperbolic genus-two surface, one of the isoperimetric regions must be an annulus.

In a planar annulus bounded by two circles, we show that the leastperimeter way to enclose a given area is an arc against the outer boundary or a pair of spokes. We generalize this result to spherical and hyperbolic surfaces bounded by circles, horocycles, and other constant-curvature curves. In one case the solution alternates back and forth between two types, a phenomenon we have yet to see in the literature.

We also examine non-orientable surfaces such as spherical Möbius bands and hyperbolic twisted chimney spaces.
\end{abstract}

\section{Introduction}

Suppose we want to enclose given area on a surface with minimum perimeter, that is, to fence off a certain amount of land using as little fencing as possible. What shape should the fence be? The isoperimetric problem, as this riddle is known, has fascinated mathematicians and laymen for millennia. As early as 200 B. C. E., Greek geometer Zenodorus showed that a circle is the solution in the plane. The problem appears repeatedly in Greco-Roman and British mythology from The Aeneid by Virgil to the thirteenth century Histories of the Kings of Britain by Geoffrey of Monmouth [14]. Jacob Steiner began the first modern rigorous proof in 1842, though it took several decades for mathematicians to prove that a solution must exist. Since Steiner, many other proofs have been found in $\mathbb{R}^{2}$ and the problem has also been solved on the cylinder, cone, hyperbolic plane, flat torus, paraboloid, and a few surfaces with density $[6$, p. 3]. This article considers this age-old problem on surfaces for which it had yet to be solved, including hyperbolic genus-two surfaces, annuli bounded

Received August 19, 2010.

2010 Mathematics Subject Classification. 53C99, 49Q99, 53A99, 30F99.

Key words and phrases. isoperimetric problem, hyperbolic surface, mobius band. 

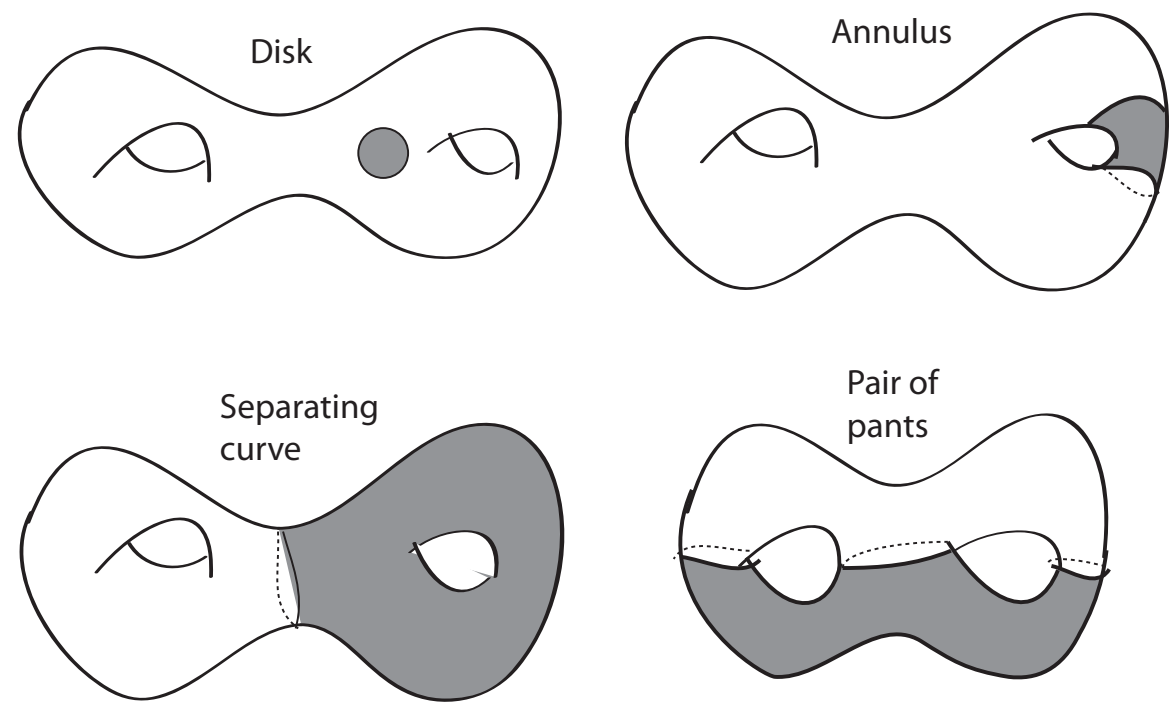

FiguRE 1. Every genus-two surface has isoperimetric disks and annuli. In some genus-two surfaces, separating curves or pairs of pants are also minimizing.

by constant-curvature curves, Möbius bands, and hyperbolic twisted-chimney spaces. We recently learned that the case of a region between two parallel horocycles was done by Marcio Silva [11].

1.1 Hyperbolic genus-two surfaces. In Theorem 5.23 we show that on every hyperbolic genus-two surface, one of the minimizers must be an annulus. Theorem 5.13 shows that every minimizer must be one of the types shown in Figure 1. Theorem 5.18 shows that each of these types actually occurs for some hyperbolic metric. Combining these results, Theorem 5.24 shows the complete set of minimizers on a given genus-two surface must be one of the following:

(1) disks and annuli,

(2) disks, annuli, and pairs of pants,

(3) disks, annuli, and separating curves,

(4) disks, annuli, separating curves and pairs of pants.

Of these, types (1)-(3) are known to actually occur.

To arrive at these results, we begin with a list of possible hyperbolic minimizers from Adams and Morgan [1, Theorems 2.2, 2.3] and see which of them can occur on a genus-two surface (Theorem 5.13). Adams and Morgan showed that disks and annuli occur on the maximal genus-two surface, so we sought to find limiting cases for which separating curves and pairs of pants occur as well. We construct these limiting cases as follows: 


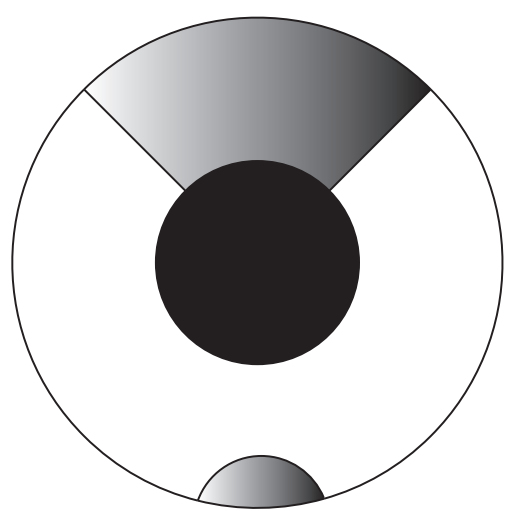

FiguRE 2. In a concentric annulus a minimizer is an arc normal to the outer boundary for small areas and a pair of spokes for large areas.

- cut the maximal genus-two surface along any three disjoint closed geodesics to get two pairs of pants;

- cut the pairs of pants into four right-angled hexagons;

- scale these hexagons so that each has three alternating sides of length $\varepsilon>0$

- reglue the hexagons to form two pairs of pants with very short cuff lengths. Depending how we attach the pairs of pants to each other we get a genus-two surface with either a very short separating geodesic or three very short non-separating geodesics. In the former case, a separating curve is minimizing for half the area of the surface, while in the latter case, a pair of pants is superior.

1.2 Regions of $\mathbb{R}^{2}, \mathbb{S}^{2}$, and $\mathbb{H}^{2}$ bounded by constant-curvature curves. In a planar annulus bounded by two concentric circles, Theorem 3.10 shows that the minimizer is an arc normal to the outer boundary for small areas and a pair of spokes for large areas as in Figure 2. This result is actually a special case of Theorem 3.9, which generalizes the solution to all connected regions $\mathbb{R}^{2}, \mathbb{S}^{2}$, and $\mathbb{H}^{2}$ bounded by two disjoint constant-curvature curves. In some of these regions, such as non-concentric annuli, spokes may be curved and vary in length. Theorem 3.7 shows that in most regions of variable width, there are exactly four spokes with a given curvature, two long spokes and two short spokes. Building on this result, Theorem 3.9 states that a minimizer must be one of the types shown in Figure 3.

The key to proving Theorem 3.9 is to show that a pair of long spokes such as those in Figure 4 are never minimizing. Take two long spokes of curvature $\kappa$ and move one spoke outward, adding perimeter $\Delta P_{1}$ and area $\Delta A$. Move the other spoke inward, saving perimeter $\Delta P_{2}$ and area $\Delta A$, thereby keeping 


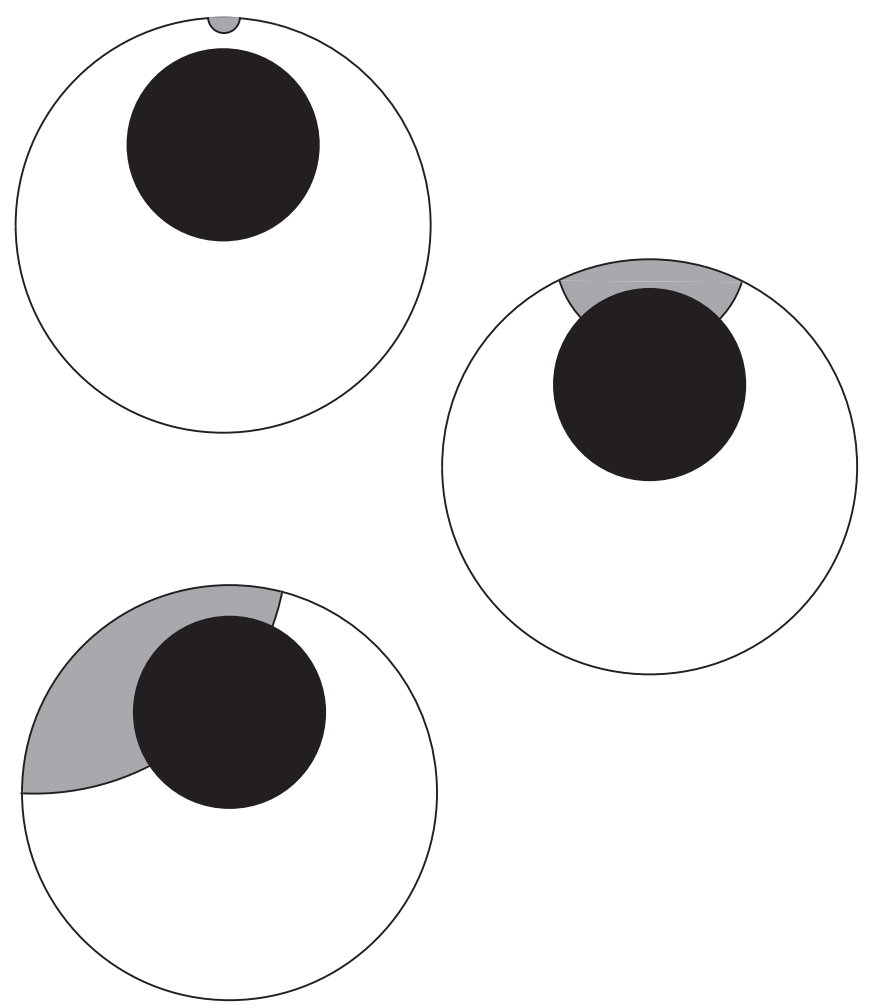

FIgURE 3. In a non-concentric annulus $S$, or almost any region bounded by two constant-curvature curves, the minimizer is an arc normal to the boundary for small areas, a pair of short spokes for medium areas, and a short and long spoke for areas close to $($ area $S) / 2$.

the total area constant. Curvature, of course, represents change in perimeter over change in area. Since the spoke we lengthened has less curvature and the spoke we shortened has more, $\Delta P_{1} / \Delta A<\Delta P_{2} / \Delta A$. Hence $\Delta P_{1}<\Delta P_{2}$, which means we have lost more perimeter than we have added. Therefore, since two deformed spokes enclose the same area as a pair of long spokes with less perimeter, two long spokes are not minimizing. By existence and regularity Theorem 2.1, the only other choices for minimizers are an arc normal to a boundary, two short spokes, or a short spoke and a long spoke of the same curvature.

1.3 Bands and related surfaces. Theorem 4.2 shows that in a band, such as those in Figure 5, a minimizer is one of the following:

(1) an arc against the boundary with less curvature,

(2) a pair of spokes, 


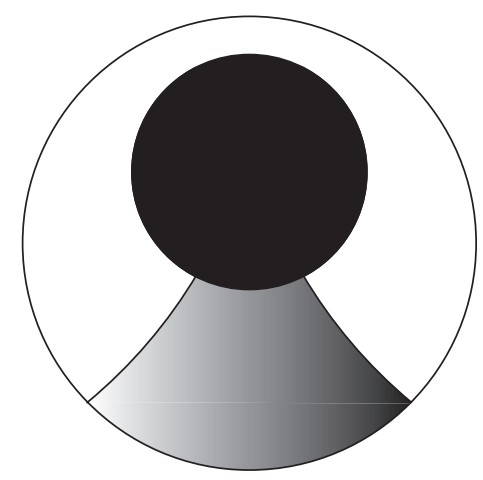

Figure 4. A non-concentric annulus with two long spokes. Although a pair of long spokes looks minimizing, we can actually save perimeter by moving one spoke up and the other down, thereby capturing the same area with less perimeter.

(3) a curve at fixed distance from a boundary, or

(4) two curves at fixed distance from a boundary.

We define a band to be a Euclidean, spherical, or hyperbolic annulus of constant width with constant-curvature boundaries. Unlike the other surfaces we study, there is no fixed order in which the minimizers occur: on some bands the minimizer is a pair of spokes for medium areas and a fixed-distance curve for large areas, while on other bands the order is reversed. In Theorem 4.4, we even find a hyperbolic band for which the minimizer is first a pair of spokes, then a fixed-distance curve, and then a pair of spokes again before we have reached half the area. This unexpected alternation between two types of minimizers is something we have yet to see in the literature.

On a Möbius band, in contrast, Theorem 4.6 shows that the order of the minimizers is always the same: an arc normal to the boundary for small areas, a fixed-distance curve for medium areas, and spokes for large areas. Propositions 4.14-4.19 show that the Euclidean band, torus, and Klein bottle isoperimetric problems are equivalent, and that we can use our list of minimizers in a band to find all the minimizers on a Euclidean strip, cylinder, or twisted chimney space.

To show that a minimizer cannot contain both an arc normal to the boundary and a fixed-distance curve, we employ the same variational argument discussed in Section 1.2.

\section{Existence and regularity}

Theorem 2.1. Let $S$ be a complete, smooth Riemannian surface, possibly with boundary. If $S$ has finite area or is compact under its isometries, then for 


\section{Fixed-distance}

Two fixed-

curve
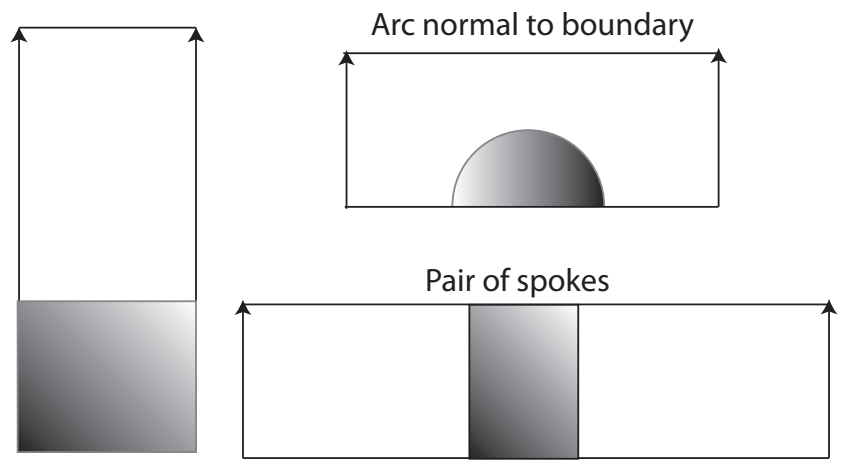

distance

curves

FiguRE 5. Four types of minimizers occur on bands: arcs normal to the boundary with more curvature, pairs of geodesic spokes, and one or two curves at fixed distance from a boundary. The band on the right is hyperbolic.

any area $0<A<$ area $S$ there exists a minimizer. It consists of curves of equal constant curvature and is smooth on the interior of $S$. Existence and regularity also hold for infinite regions of $\mathbb{R}^{2}$ or $\mathbb{H}^{2}$ bounded by one or two constant-curvature curves.

Joel Hass and Frank Morgan prove existence and regularity for smooth closed Riemannian surfaces [5, Theorem 3.4]. Geometric measure theory provides a proof to cover all $n$-dimensional manifolds that are of finite volume or are compact under their isometries [7, Theorem 2.1], [9, pp. 129-131]. To extend the theorem to infinite regions of $\mathbb{R}^{2}$ or $\mathbb{H}^{2}$ bounded by constant-curvature curves, draw a disk encompassing a large portion of the region. Since the intersection of the disk and the region has finite area, a minimizer exists. One can then prove that the minimizer in the intersection is indeed the minimizer in the entire region by taking piece of curves and translating them back into the disk.

Although Theorem 2.1 holds for all areas, on finite surfaces we will only consider areas up to (area $S$ )/2 since we can find the minimizer for larger areas by taking complements. For instance, in the flat torus, if a circle enclosing a disk is the minimizer for $10 \%$ of the area, then a circle enclosing the complement of a disk is the minimizer for $90 \%$ of the area. Every surface we examine in this paper is covered by this theorem, except for twisted chimney spaces (Corollary 4.13) and the surfaces in Section 5, where our proofs do not depend on existence and regularity. 
Remark 2.2. All boundaries discussed in this paper are "free", meaning they can be used to help enclose area without adding to the total perimeter. Therefore, if a minimizer intersects a boundary, it must do so smoothly and orthogonally at isolated points.

This result is well known in more general cases, including some manifolds of higher dimension, thanks to geometric measure theory. The proof in Riemannian surfaces involves a variational argument in which we trim off a corner and recover the lost area elsewhere by deforming the curve.

\section{Regions of $\mathbb{R}^{2}, \mathbb{S}^{2}$ and $\mathbb{H}^{2}$ bounded by constant-curvature curves}

In The Aeneid, Roman author Virgil recounts the legend of Queen Dido, who fled Tyre to escape her murderous brother Pygmalion. Arriving on the North African coast, the local leaders agreed to sell her as much beach-front as could be enclosed by a bull's hide. The clever Dido cut the bull's hide into strips making a very long rope which she strung in a semi-circle perpendicular to the coast, thereby capturing as much land as possible. Queen Dido's geometric insight not only led to the founding of Carthage, but is also one of the earliest examples of the challenge we are about to explore: finding the least-perimeter way to enclose a given area in a region with free boundary [14].

Lemma 3.1. In a region of $\mathbb{R}^{2}, \mathbb{S}^{2}$ or $\mathbb{H}^{2}$ bounded by one constant-curvature curve, the minimizer is a constant-curvature curve normal to the boundary. Conversely, any constant-curvature curve normal to the boundary at its endpoints minimizes length for a given area, uniquely up to translation along the boundary.

Proof. By Theorem 2.1, a minimizer must have constant curvature. Suppose a minimizer contains a component which does not intersect the boundary. Since a minimizer must have finite length, this component must be a circle, and we can translate it so that it is tangent to the boundary or another component contradicting regularity. Thus, all components intersect the boundary and must do so twice since they have finite length. Multiple components can be translated along the boundary until they touch, again violating regularity. Thus, the minimizer consists of a single constant-curvature curve normal to the boundary.

To show uniqueness, suppose that there are distinct constant-curvature curves of length $L$ and enclosing area $A$ both normal to the boundary. By translation, we can assume the two curves have a common right endpoint. Since distinct, they must have different curvatures. Since they enclose the same area, the one with greater curvature must return later, contradicting Schur's lemma $[2$, p. 46] as shown in Figure 6.

Every constant-curvature curve normal to the boundary at both endpoints is determined, up to translation, by its curvature. The greater the curvature, the greater the area enclosed by the curve. Suppose under contradiction, that there exists such a curve $C$ enclosing area $A$ which in not minimizing. By 


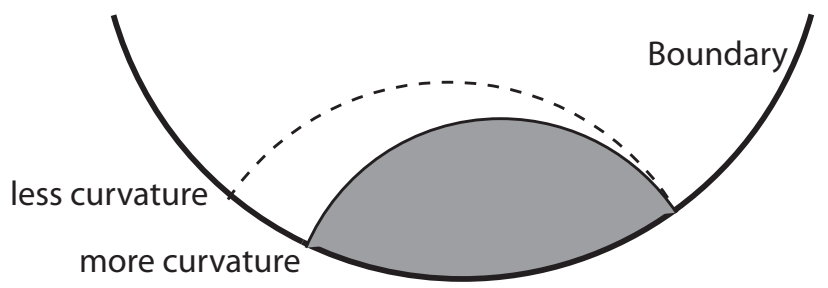

Figure 6. Curves with different curvatures enclose different areas.

existence, we know there must be a minimizer for area $A$ and we just showed that it must be a constant-curvature curve normal to the boundary. Since the minimizer is not congruent to $C$, it must have different curvature. However, if it has a different curvature, it must enclose a different amount of area, contradiction. Therefore, every constant-curvature curve normal to the boundary at its endpoints is a minimizer.

We pause briefly to note that there is only one type of constant-curvature curve in the sphere (circles), two types in the Euclidean plane (lines and circles) and three types in the hyperbolic plane (circles, horocycles, and equidistant curves).

Definition 3.2. An equidistant curve is a curve at constant distance from a geodesic.

Note that all geodesics are equidistant curves since they lie at constant distance from themselves.

In the sphere, all circles are equidistant curves. The Arctic and Antarctic circles, for example, are equidistant from the equator. Every spherical geodesic has exactly two equidistant curves of every curvature, one on each side. In the Euclidean plane, the only equidistant curves are lines. In the hyperbolic plane, however, space spreads out so quickly that a curve can have non-zero curvature and yet never close up to form a circle. With the exception of horocycles, all curves with this interesting property are equidistant curves. Around every hyperbolic geodesic, there are two equidistant curves of curvature $0<\kappa<1$, one on each side. The geodesic in this case is called the axis. Equidistant curves very close to the axis will be almost as straight as geodesics, but the farther out you go, the greater the curvature of the equidistant curves. Needless to say, all equidistant curves in the surfaces we discuss must have constant curvature.

Remark 3.3. Let $C$ be a constant-curvature curve in the hyperbolic plane with curvature $\kappa$. If $|\kappa|>1, C$ is a circle. If $|\kappa|=1, C$ is a horocycle. If $|\kappa|<1, C$ is an equidistant curve.

In the half-plane model of $\mathbb{H}^{2}$ where geodesics are either semi-circles or vertical lines, equidistant curves are represented by circular arcs or diagonal 


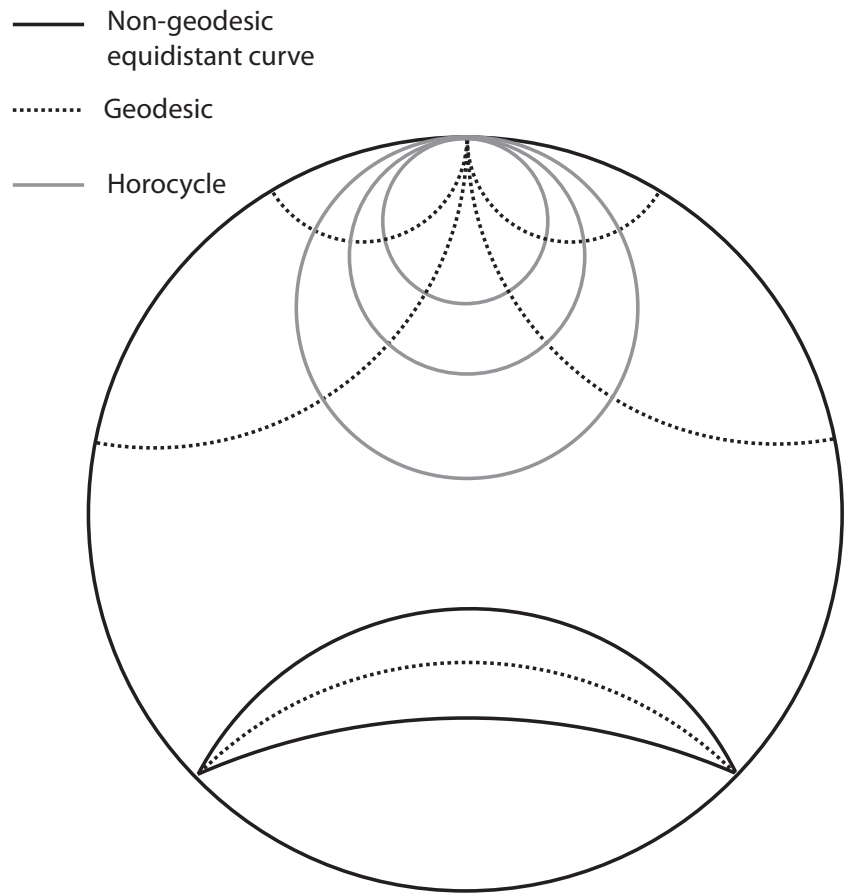

Figure 7. Constant-curvature curves in the Poincaré disk model of $\mathbb{H}^{2}$.

lines, intersecting their axes at the boundary. Horocycles appear as horizontal lines or circles tangent to the boundary. In the Poincaré disk model (Figure 7) where geodesics are represented by circular arcs normal to the boundary, equidistant curves are represented by circular arcs intersecting their axes at the boundary. Horocycles appear as circles tangent the boundary. In either model, horocycles that touch the boundary at the same point are at constant distance from one another.

Having dealt with regions bounded by one constant-curvature curve, we shall now look at connected regions bounded by two, including annuli, the complement of a disk in the half plane, the complement of two disks in the plane or sphere, and infinite strips. In any of these regions, the minimizer for small areas will clearly be a constant-curvature curve normal to one of the boundaries. But which boundary should we choose? The following lemma shows that we must always pick the boundary with greater curvature.

Lemma 3.4. Take two copies of the Euclidean plane, the sphere, or the hyperbolic plane. Consider the portion of each copy bounded by a constant-curvature curve. If the two boundaries have different curvatures, then a constant-curvature curve normal to the boundary with greater curvature will enclose more area 

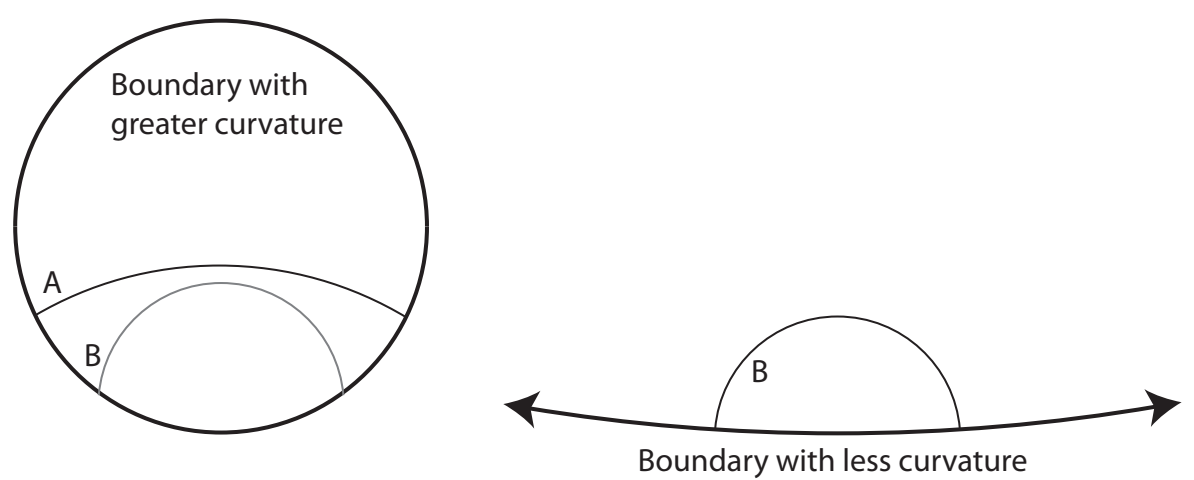

FiguRE 8. A constant-curvature curve normal to the boundary with greater curvature (A) is always superior to a curve of the same length against the boundary with less curvature (B).

than a constant-curvature curve of the same length normal to the boundary with less curvature. Convex boundaries are assumed to have negative curvature.

Proof. Let $A$ be a constant-curvature curve normal to the boundary with greater curvature and $B$ be a constant-curvature curve of the same length normal to the boundary with less curvature. Compare curves $A$ and $B$ when placed against the boundary with greater curvature as in Figure 8. Since $A$ is normal to the boundary, then by Lemma 3.1 it is the unique minimizer in that surface, and hence encloses more area than $B$. However, $B$ encloses more area against the boundary with more curvature than it does against the boundary with less curvature as shown in Figure 8. Thus, $A$ encloses more area against boundary the boundary with greater curvature than $B$ does against the boundary with less curvature.

In surfaces with two boundaries, we can expect some minimizers to go from one boundary to the other. In an annulus, these curves look like spokes on a wheel.

Definition 3.5. In a region between two constant-curvature curves, any constant-curvature curve that meets both boundaries orthogonally is called a spoke.

If the boundaries are at a constant distance from one another all spokes will be geodesics and have the same length. However, if the distance between the boundaries varies, the length and curvature of the spokes will too. In fact, every spoke is really just an arc of a circle (or some other constant-curvature curve) which meets both boundaries orthogonally.

Definition 3.6. A pair of spokes with maximal curvature are called maximal spokes. Spokes longer than the maximal spokes are called long spokes; spokes 


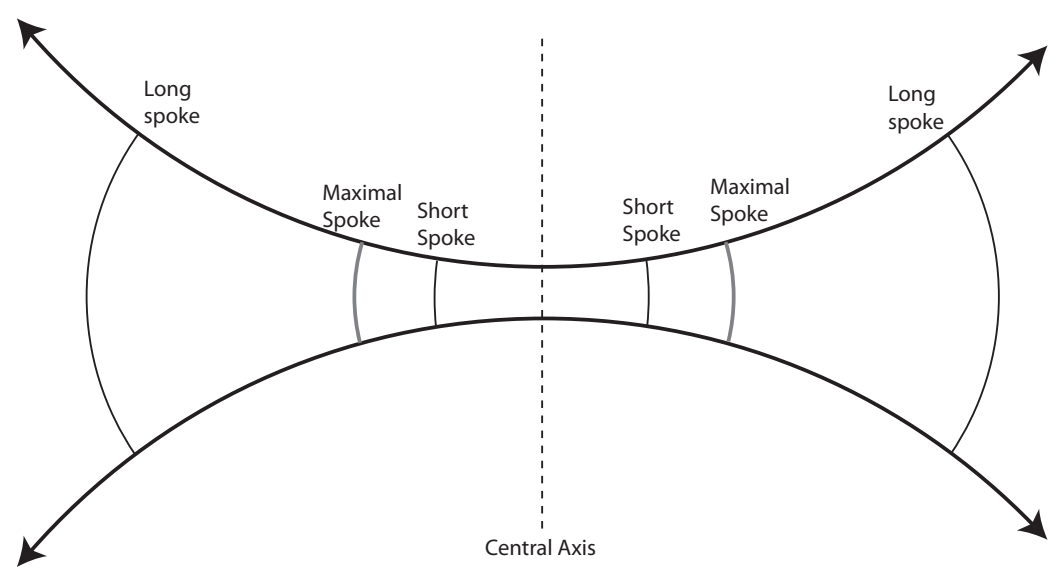

FiguRE 9. A spoke is a constant-curvature curve that meets both boundaries orthogonally.
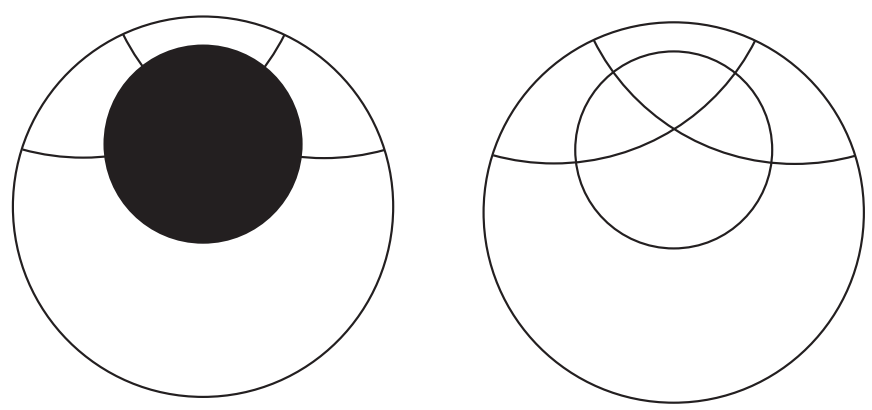

Figure 10. At left, a non-concentric annulus with four spokes of the same curvature, two long and two short. At right, we observe that such spokes are formed from the intersection of the annulus with two identical circles.

equal to or shorter than the maximal spokes are called short spokes (including the maximal spokes themselves). A geodesic axis that crosses both boundaries orthogonally at the points where they are closest is called the central axis (see Figure 9).

Of course, in a region where the two boundaries meet at infinity, no central axis exists since the boundaries never reach a closest point. All other regions bounded by two constant-curvature curves have a central axis, however, and most have maximal spokes. 
Lemma 3.7. Consider a region of $\mathbb{R}^{2}, \mathbb{S}^{2}$, or $\mathbb{H}^{2}$ between two constantcurvature curves whose width is not constant. If the region does not contain maximal spokes, then spoke curvature increases monotonically in either direction starting at the central axis. If the region does contains maximal spokes,

(1) There exists a unique pair of maximal spokes. The two spokes have the same length, are reflections of one another across the central axis, and are arcs of the same constant-curvature curve.

(2) As we move away from a maximal spoke in either direction, spoke curvature decreases monotonically until it reaches 0 (if ever).

(3) Given any curvature $0<\kappa<\kappa_{\max }$, there exist exactly four spokes with that curvature: two long spokes of the same length, and two short spokes of the same length (see Figure 10).

In $\mathbb{R}^{2}$ and $\mathbb{S}^{2}$ maximal spokes always occur.

Proof. Consider a region of $\mathbb{R}^{2}, \mathbb{S}^{2}$, or $\mathbb{H}^{2}$ between two constant-curvature curves, $A$ and $B$, whose width is not constant. If the region is in $\mathbb{H}^{2}$, picture it in the Poincaré disk model. Draw a small circle that intersects boundary $A$ orthogonally centered on the central axis. Enlarge the circle, keeping it orthogonal to $A$, until it meets $B$ orthogonally as well (see Figure 11). For hyperbolic regions, the circle might not be contained in the Poincaré disk, as in Figure 12, and hence may represent a horocycle or equidistant curve. Call this "circle" $M$ and its curvature $\kappa_{m}$. Note that $M$ generates two spokes of equal length and curvature.

(1) Recall that the two boundaries come closest at the central axis. As we travel away from the central axis along one of the boundaries, the other boundary gets steadily father away. Therefore, if we slide $M$ along boundary $A$ in either direction, keeping it orthogonal to $A$, the centers of $M$ and $B$ will become farther apart. As a result, the angle of intersection between $M$ and $B$ will decrease, as shown in Figure 13. Therefore, enlarge $M$ to create a new circle that is once again orthogonal to both boundaries. Since this new circle is larger than $M$, the spokes it generates will have less curvature than the spokes generated by $M$. Therefore, the spokes obtained from $M$ have maximal curvature.

(2) The farther we translate $M$, the farther apart $A$ and $B$ will become and hence the more we will have to decrease the curvature. Therefore, spoke curvature decreases monotonically in both directions, approaching zero. By symmetry, spoke curvature reaches zero at the central axis. For hyperbolic regions, if the maximal spokes are not contained within the Poincaré disk, then maximal spokes never occur. Therefore, the curvature goes on increasing forever as we move away from the central axis.

(3) The two maximal spokes and central axis divide our surface into four regions. Note that within each region, spoke curvature must vary monotonically between 0 and $\kappa_{m}$. Therefore, given any curvature $0<\kappa<\kappa_{m}$, there exist exactly four spokes with that curvature. Clearly, the two regions inside of $M$ 


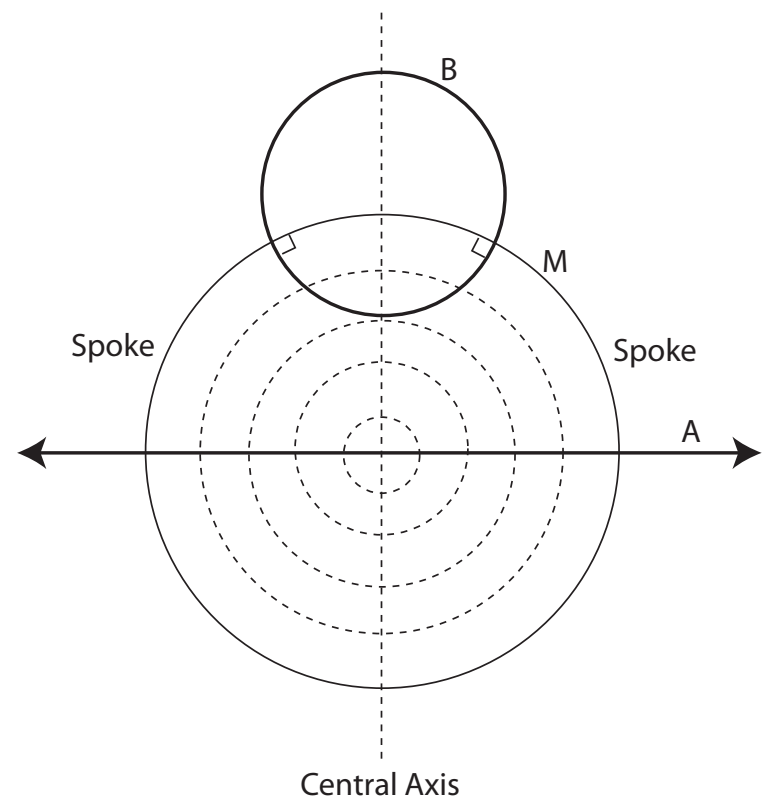

Figure 11. Begin with the smallest dashed circle, centered on the central axis. Enlarge it until it is orthogonal to both boundaries. By definition, the arcs of this circle connecting the boundaries are spokes.

contain short spokes, while two outside contain long spokes. Thus, for every curvature $0<\kappa<\kappa_{m}$, there exist two long spokes and two short spokes.

As we have seen, not all regions have a unique pair of maximal spokes. In surfaces of constant width, such as a Euclidean strip or a concentric annulus, all spokes are geodesics of the same length. In hyperbolic strips, spoke curvature increases as we move away from the central axis, never reaching a maximum. A more interesting example is a region between two horocycles that approach the same point at infinity in the hyperbolic plane. In this region, all spokes are geodesics $(\kappa=0)$. Similarly, in a region between two hyperbolic geodesics that approach the same point, all the spokes are horocycles $(\kappa=1)$. In either of these surfaces, a single spoke can bound any area.

Remark 3.8. Although maximal spokes do not occur on all hyperbolic regions, they seem to occur if and only if a region has non-constant width and the sum of its boundary curvatures is negative. For instance, in the region between two disks, the boundaries both have negative curvature so maximal spokes occur. In the region between two geodesics, the sum of the curvatures is 0 , so maximal spokes do not occur. A rigorous proof of this proposition would be a interesting topic for further research. 


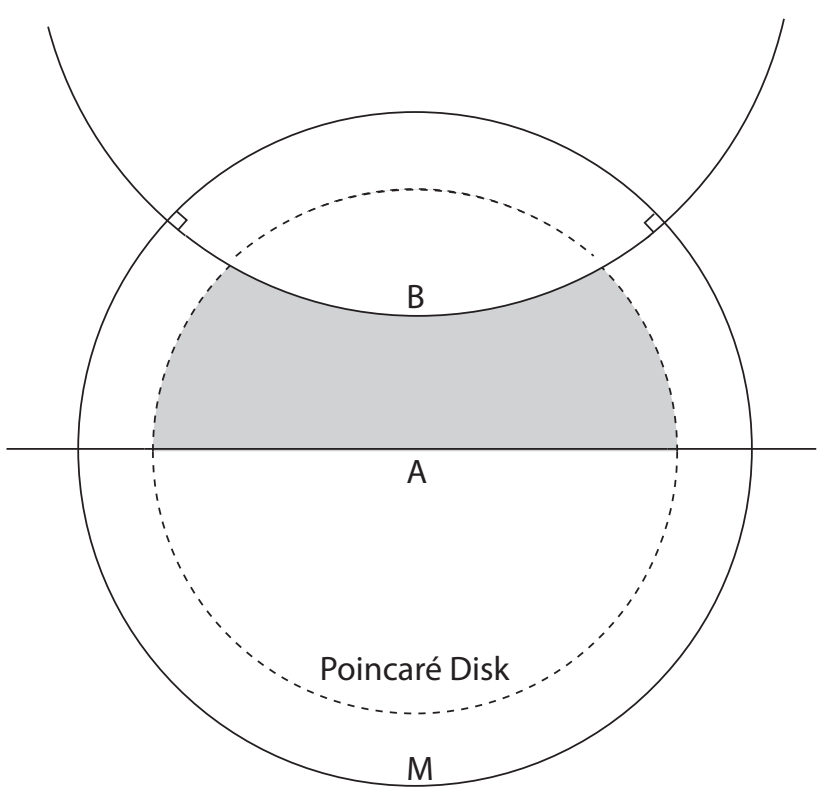

FiguRE 12. If the region is hyperbolic, $M$ might not be contained within the Poincaré disk. In that case, spoke curvature never reaches a maximum.

Now at last we are ready to solve the isoperimetric problem in a region bounded by two constant-curvature curves, the main result of this section.

Theorem 3.9. Let $S$ be a region of $\mathbb{R}^{2}, \mathbb{S}^{2}$ or $\mathbb{H}^{2}$ between two constantcurvature curves. If the boundaries approach the same point at infinity in the hyperbolic plane, the minimizer is a spoke bounding a cusp (see Figure 14). Otherwise, there exist $0<A_{1}<A_{2} \leq($ area $S) / 2$ such that a minimizer is

(1) a constant-curvature curve normal to the boundary with greater curvature for $A \leq A_{1}$,

(2) two short spokes with equal curvature for $A_{1} \leq A \leq A_{2}$,

(3) a short spoke and a long spoke with equal curvature for $A_{2}<A \leq$ $($ area $S) / 2$.

(1) and (2) always occur, but in certain infinite hyperbolic regions and in regions of constant width (3) does not occur.

Proof. By Theorem 2.1, a minimizer consists of constant-curvature curves. If the two boundaries approach the same point at infinity then the surface has a cusp. For the first part of the proof, assume $S$ has no cusps.

Suppose some component of a minimizer intersects no boundaries. Since it must have finite length, it has to be a circle. However, circles can be ruled out 

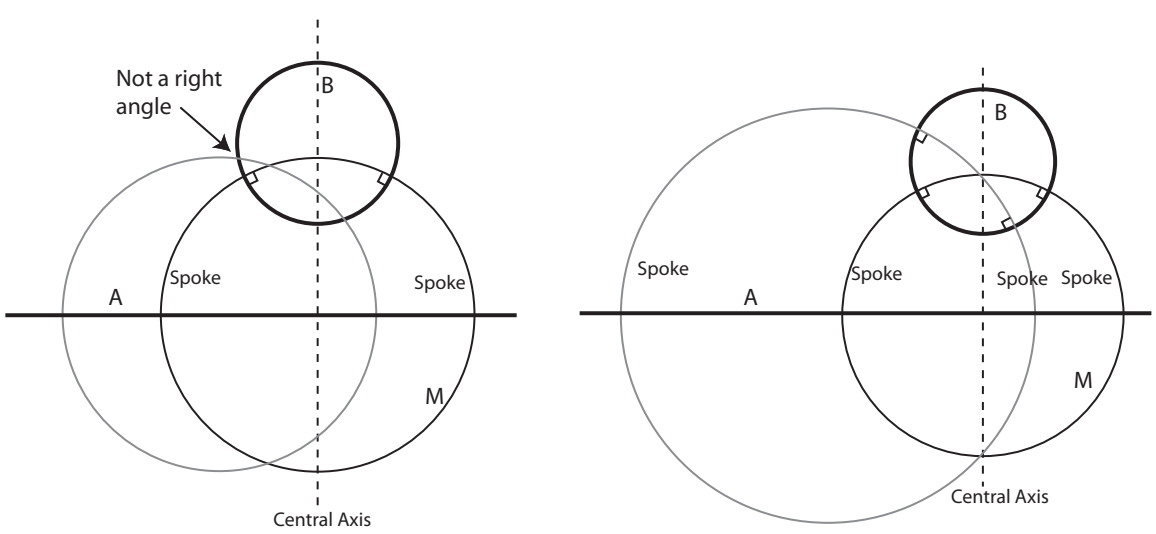

FiguRE 13. When we translate a copy of $M$ along boundary $A$, it will no longer meet boundary $B$ orthogonally. Therefore, we must enlarge the copy until it meets both boundaries orthogonally. The resulting spokes have less curvature, so the original spokes generated by $M$ must have maximal curvature.

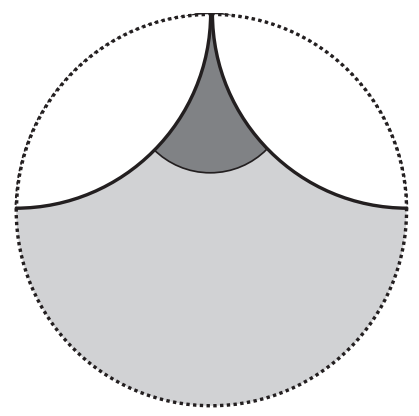

FiguRE 14. An infinite hyperbolic region shown in the Poincaré disk model. Since the boundaries approach the same point at infinity, the minimizer is a spoke bounding a cusp.

since we can translate them so that are tangent to the boundary or another component, contradicting regularity.

Suppose that some component of a minimizer intersects both boundaries. Since it must intersect the boundaries orthogonally, it is a spoke. The minimizer cannot contain a component touching only one boundary, since such a component could be translated up against a spoke or another curve, contradicting regularly. Thus, the minimizer consists entirely of spokes of the same curvature.

Suppose every component intersects exactly one boundary. Therefore, it is an arc normal to a boundary. Multiple arcs against the same boundary 


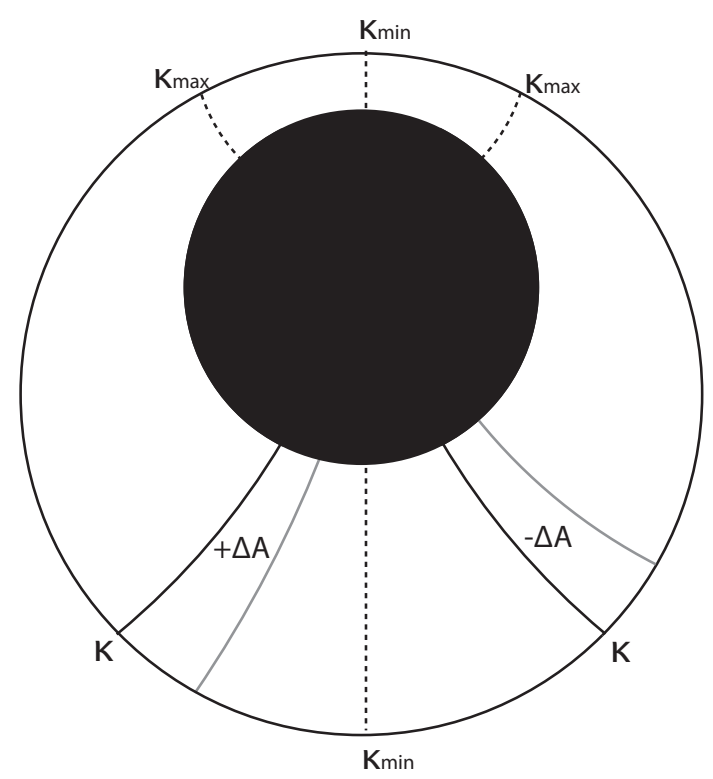

FiguRE 15. Start with two long spokes (black) and deform them into the grey spokes. The grey spokes enclose the same area as the black ones and have less perimeter. Therefore, long spokes are never minimizing.

can be translated so that they touch tangentially, contradicting smoothness. Thus, the minimizer consists of either one arc or an arc against each boundary. However, an arc against either boundary is no better than two arcs against the boundary with greater curvature (Lemma 3.4), which we have already ruled out. Note that it will always be possible to draw a second arc on the boundary with greater curvature without bumping the other boundary, because arcs against the boundary with greater curvature are not as tall as those against the boundary with less curvature. Thus, the minimizer is a single arc normal to the boundary with greater curvature.

If the boundaries approach the same point at infinity forming a cusp, then we can rule out arcs by translating them out along the cusp until they bump the other boundary. One spoke bounding a cusp is clearly shorter than two arcs bounding the same area, since spokes farther from the cusp are longer. Therefore, the minimizer for any area is a spoke bounding a cusp.

Henceforth, assume the boundaries do not approach the same point at infinity. Clearly, arcs are always minimizing for the smallest areas since there is no lower bound on their perimeter. However, arcs cannot be the minimizer for every area: if the region has infinite area, then there are clearly areas too big 
for an arc to enclose. If the region has finite area, it is an annulus and any arc in an annulus that captures half the area can be translated so that it bumps the inner boundary, a contradiction. Thus, every region has arcs and spokes as minimizers.

As we can see from Figure 3, the curvature of a minimizing arc is clearly greater than the curvature of any two spokes. Therefore, once the area is large enough for spokes to be optimal, they will continue to be optimal for larger areas. Thus, there exists an area $A_{1}>0$ such that arcs against a boundary are minimizing if $A \leq A_{1}$ and spokes are minimizing for $A_{1} \leq A \leq($ area $S) / 2$.

If $S$ does not have maximal spokes, then all spokes are short by definition, so a minimizer is either an arc or a pair of short spokes. If $S$ does have a pair of maximal spokes, then for any curvature $0<\kappa<\kappa_{\max }$, there are exactly four spokes, two long and two short (Lemma 3.7). For smaller areas, a pair of short spokes is clearly best. However, short spokes can only enclose as much area as the maximal pair. For large areas, the solution is either a pair of long spokes or a long-short combination.

Suppose that a pair of long spokes with curvature $\kappa$ is minimizing (see Figure 15). Deform the left spoke left downwards, adding perimeter $\Delta P_{1}$ and area $\Delta A$. Deform the right spoke upwards, saving perimeter $\Delta P_{2}$ and area $\Delta A$. Note that the curvature of the left spoke has decreased, while the curvature of right spoke has increased. Since curvature represents change in perimeter over change in area, $\Delta P_{1} / \Delta A<\Delta P_{2} / \Delta A$ so $\Delta P_{1}<\Delta P_{2}$. Therefore, by deforming the spokes we save more perimeter than we added but enclosed the same area, contradiction. Hence, by process of elimination, the minimizer for large areas must be a long-short combination.

Let $A_{2}$ be the area enclosed by the maximal spokes. The maximal spokes clearly enclose more area than the largest minimizing arc normal to the boundary, so $A_{1}<A_{2}$. If the region has finite area, it is an annulus, and the maximal pair clearly encloses less than half the area as shown in Figure 15. Therefore $A_{1}<A_{2} \leq($ area $S) / 2$. Since the shortest long-short combination encloses more area than the maximal spokes, clearly short spokes are minimizing for $A_{1} \leq A \leq A_{2}$ and the long-short combination is minimizing for $A_{2} \leq A \leq($ area $S) / 2$.

We conclude with two special cases of Theorem 3.9.

Corollary 3.10. Let $S$ be an annulus bounded by two concentric circles. Then there exists $0<A_{0}<($ area $S) / 2$ such that the least-perimeter way to enclose area $A \leq($ area $S) / 2$ is

(1) a constant-curvature curve normal to the outer boundary if $A \leq A_{1}$,

(2) a pair of geodesic spokes if $A_{0} \leq A \leq($ area $S) / 2$.

Proof. Since the circles are concentric, the distance between them is constant. Therefore, all spokes are geodesics of the same length. The rest of the proof follows immediately from Theorem 3.9. 
The following corollary offers an alternative to Lee's elegant proof for the spherical strip [7, Theorem 3.2]. Note that annuli and strips are the same on a sphere.

Corollary 3.11. Let $S$ be a spherical strip, that is, the region on a sphere between two circles of latitude. Then there exists $0<A_{1}<($ area $S) / 2$ such that the least-perimeter way to enclose area $A \leq($ area $S) / 2$ is

(1) a circular arc normal to the latitude closer to the equator if $A \leq A_{1}$,

(2) two arcs of longitude if $A_{1} \leq A \leq($ area $S) / 2$.

Proof. Latitudes closer to the equator have less curvature. Arcs of longitude are the only constant-curvature curves that cross multiple latitudes perpendicularly. The rest of the proof follows immediately from Theorem 3.9.

\section{Bands and related surfaces}

We now move from regions of the plane to other constant-curvature surfaces bounded by constant-curvature curves. For instance, what is the most efficient way to enclose area on the surface of an ordinary rubber band?

Definition 4.1. A band is a constant-curvature annulus of constant width bounded by constant-curvature curves. The shortest distance around a band is called the circumference. A fixed-distance curve is a closed curve at fixed distance from a boundary, as in Figures 5 and 16.

To form a spherical strip, peel off a strip of a sphere between two latitudes, cut it along two longitudes, and identify the ends. The strip can wrap around the sphere $t$ times $(0<t<\infty)$ in order to give us a band of any finite length. To form a hyperbolic band, consider the region of the hyperbolic plane bounded by two concentric circles, two horocycles at fixed distance from one another, or two equidistant curves sharing a common axis. Since all the spokes between these boundaries are geodesics and have the same length, cut along two spokes and identify them to get a hyperbolic band. Note that the definition of a band includes a concentric circular annulus.

Given its Gauss curvature, a band is determined by three parameters: the width, the circumference, and the curvature of one of the boundaries. The curvature of the other boundary can be substituted for either of the first two parameters. Note that the boundaries need not have the same curvature or the same length. Since the distance between two spherical or hyperbolic geodesics is never constant, a band with two geodesic boundaries must be Euclidean. The following theorem characterizes all minimizers on a band. See Figure 5 for illustration.

Theorem 4.2. Let $S$ be a band of width $w$ and circumference $c$. Then the least-perimeter way to enclose area $A \leq($ area $S) / 2$ is

(1) an arc normal to the boundary with less curvature,

(2) a fixed-distance curve, 


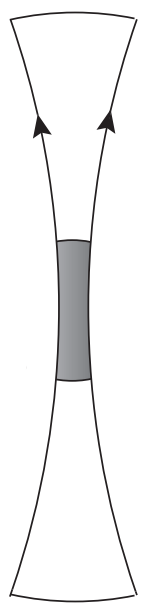

Figure 16. Two fixed-distance curves might sometimes be better than one. In this case, however, an arc normal to a boundary might be able to enclose the same area more efficiently, so it is still an open question whether two fixeddistance curves are ever minimizing.

(3) a pair of spokes, or

(4) two fixed-distance curves. At least (1)-(3) actually occur.

Proof. By Theorem 2.1, minimizers exist and consist of constant-curvature curves.

Suppose some component of a minimizer intersects no boundaries. Then the component is either a circle or it wraps at least once around the band. Any circle can be translated so that it is tangent to a boundary or another curve, violating regularity. Therefore, the component must wrap around the band. The component cannot wrap around multiple times without crossing itself, so it must wrap around exactly once. If the component is not at fixed distance from a boundary, than we can find a shorter curve enclosing the same area. Therefore, the component is a fixed-distance curve. Two such curves can sometimes enclose are given area more efficiently than one as shown in Figure 16 , but there is no advantage of having more than two.

If a component intersects both boundaries, then it must be a spoke. Two spokes are necessary to enclose area, but there is no advantage to be gained by having more than two. Clearly spokes cannot coexist with fixed-distance curves since they would cross, violating regularity. They also cannot coexist with a component intersecting only one boundary, since that component can be translated so that it touches one of the spokes or another curve, violating regularity. 


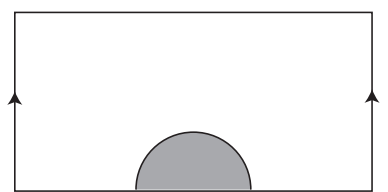

1. Arc normal to a boundary

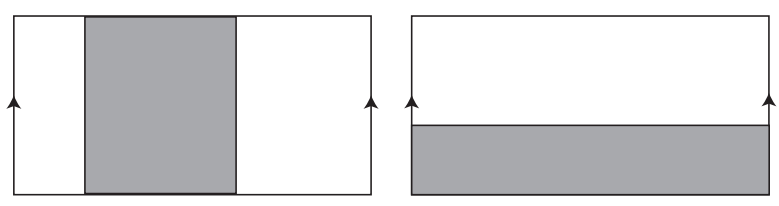

2. Spokes or Fixed-distance curve

FiguRE 17. In this Euclidean band, spokes and fixed-distance curves are equally good for large areas.

Suppose a component intersects exactly one boundary. Then it must do so twice since it has finite length and must do so orthogonally, so it is an arc. We use a variational argument, similar to 3.9 , to rule out multiple arcs as well as arcs and fixed distance curves.

In the following example, we consider a rather simple band that will serve as a jumping off point for one of the most interesting surfaces we have yet to encounter.

Lemma 4.3. Let $S$ be Euclidean band of width $w$ and circumference $2 w$ constructed from a Euclidean rectangle with the left and right sides identified. If $A \leq 2 w^{2} / \pi$, the least-perimeter way to enclose area $A$ is a semi-circle against a boundary. If $2 w^{2} / \pi \leq A \leq($ area $S) / 2$ a pair of spokes and a fixed-distance curve are both minimizing, as in Figure 17.

Proof. By Theorem 4.2 a minimizer is either an arc normal to the boundary (in this case, a semi-circle), a pair of spokes, or a fixed-distance curve (in this case a parallel line). Due to the dimensions of the band, the pair of spokes has the same length as fixed-distance curve, so they are minimizing for the same range of areas. The formula for a Euclidean semi-circle is $L^{2}=2 \pi A$, so setting $L=2 w$ we see that semi-circle only minimizing for areas below $2 w^{2} / \pi$. Since $($ area $S)=2 w^{2}$, therefore $2 w^{2} / \pi<($ area $S) / 2$, so all three types of minimizers occur.

The reader may notice that our theorem did not give a range of areas for each minimizer, as we did in Theorem 3.9. Unlike the minimizers described 


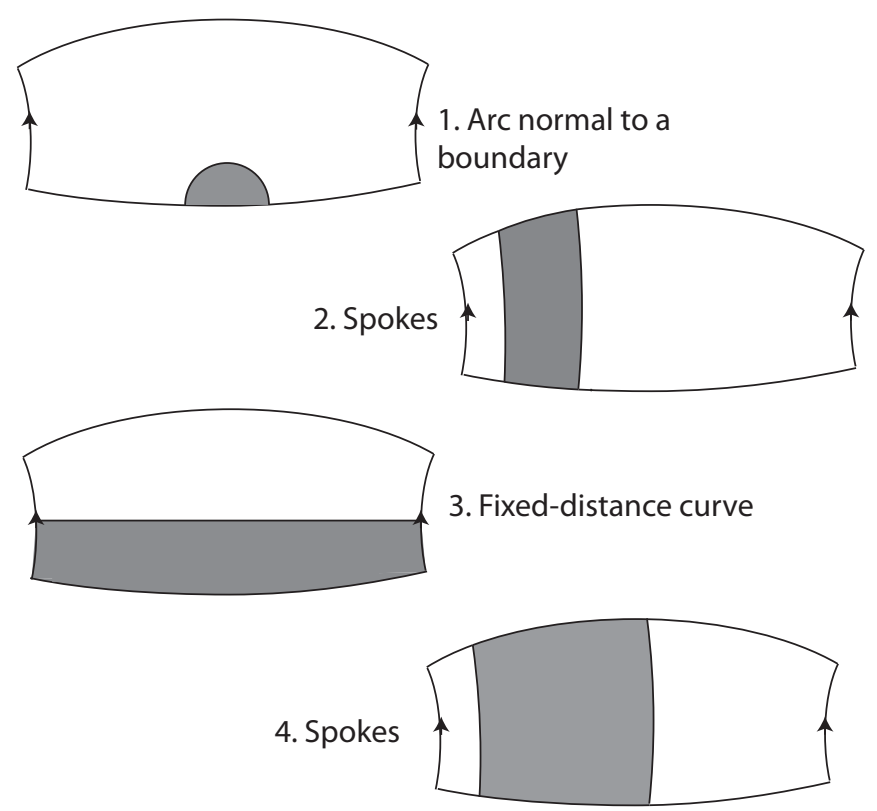

FiguRE 18. In this hyperbolic band, the minimizer alternates between two types as the enclosed area increases.

in Section 3, the order in which the minimizers occur varies depending on the parameters of the band.

Theorem 4.4. For certain hyperbolic bands, such as the one portrayed in Figure 18, there exist $0<A_{1}<A_{2}<A_{3}<($ area $S) / 2$ such that the leastperimeter way to enclose area $A$ is

(1) an arc normal to the boundary with less curvature if $A \leq A_{1}$,

(2) a pair of spokes if $A_{1} \leq A \leq A_{2}$,

(3) a fixed-distance curve if $A_{2} \leq A \leq A_{3}$,

(4) a pair of spokes if $A_{3} \leq A \leq($ area $S) / 2$.

See Figure 18 for illustration.

Proof. Let $S$ be a hyperbolic band of small width $w$ and circumference $c=2 w$ with a closed geodesic running down the middle. Therefore, the fixed-distance geodesic has the same length as a pair of spokes, but all other fixed-distance curves are slightly longer. Since small hyperbolic surfaces are nearly Euclidean, arcs normal to a boundary are best up to $A_{1} \approx 2 w^{2} / \pi<($ area $S$ ) $/ 2$ (Lemma 4.3). Spokes are the sole minimizers for larger areas, except (area $S) / 2$ where spokes and fixed-distance curves are equal. Let $A_{g}$ be the area enclosed by the fixed distance geodesic. 

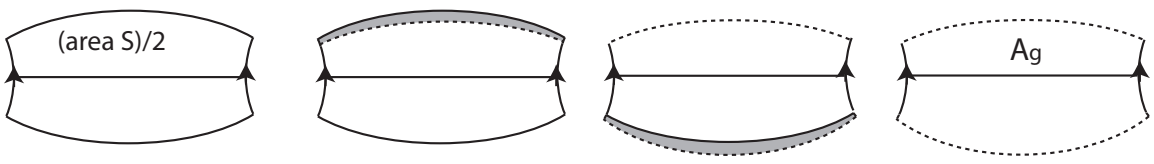

Figure 19. Begin with band in which the fixed distance geodesic runs straight down the middle, enclosing half the area. Trim the top and add to the bottom so that the fixed-distance geodesic encloses area $A_{g}$ which is less than half the area.
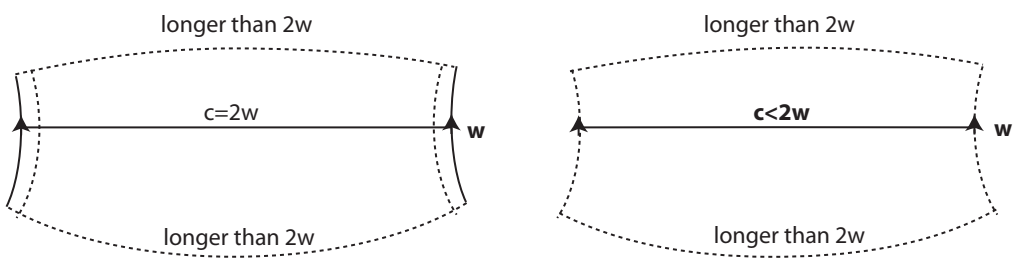

FiguRE 20. By trimming along two spokes, we can reduce the circumference just enough that a fixed-distance curve will win out for a small range of areas.

1) Trim a small amount of area of the top of the band by cutting along a fixed-distance curve, as in Figure 19. Add this area to the bottom. Now the fixed-distance geodesic encloses slightly less than half the area. Therefore, spokes and fixed-distance curves are equal at $A_{g}<($ area $S) / 2$.

2) Trim the band along two geodesic spokes to reduce the circumference very slightly, as in Figure 20. Now $c<2 w$, so the fixed-distance geodesic is actually shorter than a pair of spokes. Therefore, fixed-distance curves are superior to spokes at $A_{g}$ and nearby areas. Specifically, for some small $\varepsilon>0$, fixed-distance curves are minimizing when $A_{g}-\varepsilon<A<A_{g}+\varepsilon$. Since $c$ is only slightly less than $2 w$, therefore $A_{1}<A_{g}-\varepsilon<A_{g}+\varepsilon<($ area $S) / 2$. Setting $A_{2}=A_{g}-\varepsilon$ and $A_{3}=A_{g}+\varepsilon$ we arrive at the desired inequality.

Finally, we need to make sure no further alternation occurs. The length of a fixed-distance curve decreases monotonically as area increases from 0 to $A_{g}$, and increases monotonically after that. Therefore, fixed-distance curves are only superior for $A_{2}<A<A_{3}$. Likewise, once the area enclosed exceeds $A_{1}$, arcs normal to the boundaries get longer and longer while spokes stay the same length. Therefore, arcs normal to the boundaries are only minimizing for $A<A_{1}$. The length of a pair of spokes is constant, so spoke are only minimizing for $A_{1}<A<A_{2}$ and $A_{3}<A<($ area $S) / 2$. Therefore, no further alternation occurs.

We'll now see that the unusual alternation of Theorem 4.4 never occurs on a Möbius band. 

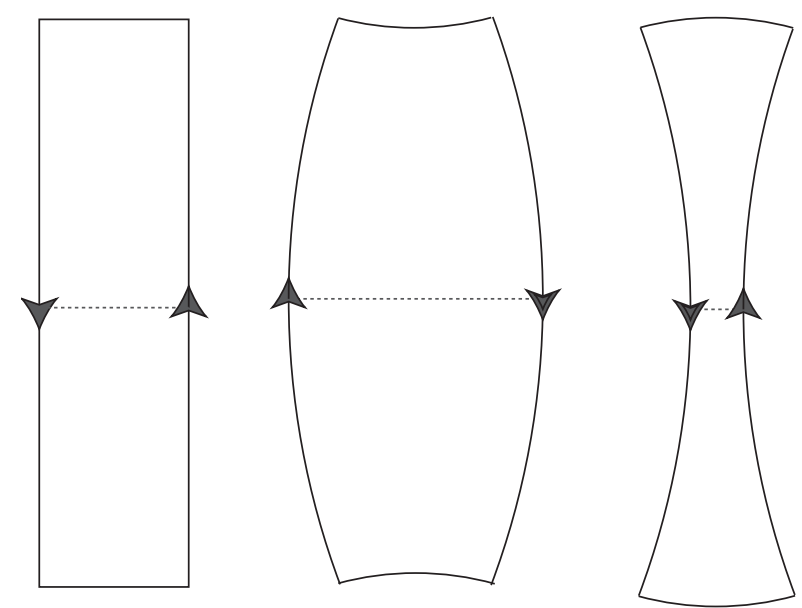

FIGURE 21. Euclidean, spherical, and hyperbolic Möbius bands each have a closed geodesic running down the middle.

Remark 4.5. Every Möbius band contains a fixed-distance geodesic running down the center, which of course encloses no area. The fixed distance curve that encloses the most area lies halfway between the middle and the boundary. Therefore, on a hyperbolic or spherical Möbius band, the length of a fixeddistance curve increases with the area enclosed (see Figure 21).

Theorem 4.6. Let $S$ be a Möbius band. Then there exist $A_{1} \leq A_{2} \leq($ area $S) / 2$ such that a minimizer is

(1) an arc normal to the boundary for $A<A_{1}$,

(2) a fixed-distance curve for $A_{1}<A<A_{2}$,

(3) a pair of spokes for $A_{2}<A<($ area $S) / 2$.

On some bands, fixed-distance curves do not occur, on some bands spokes do not occur, and on some bands, neither occur.

Proof. The argument for what types of minimizers are possible is the same for as Theorem 4.2. We now examine the order in which they occur.

An arc normal to the boundary is the only minimizer with no lower bound on its perimeter, so it is clearly best for small areas. Hence, there exists $A_{1}$ such that for area $A<A_{1}$ arcs normal to the boundaries are minimizing. In a hyperbolic or spherical Möbius band, the length of a fixed-distance curve will get longer and longer as the enclosed area increases (Remark 4.5). The length of a pair of spokes, however, is constant. Therefore, there exists $A_{2}$ such that a fixed-distance curve is minimizing for area $A_{1}<A<A_{2}$ and a pair of spokes is minimizing for area $A_{2}<A \leq($ area $S) / 2$. Clearly spokes do not occur in Möbius bands with a very small circumference while fixed-distance curves do not occur in Möbius bands with a very large circumference such as 

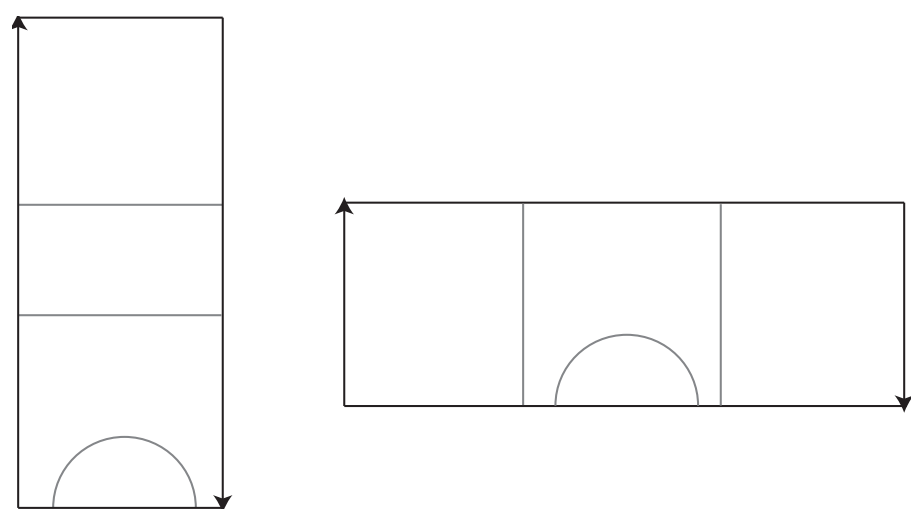

Figure 22. In a Euclidean Möbius band with a small circumference (relative to the width) the minimizers are arcs normal to the boundaries and fixed-distance curves. If the circumference is large, the minimizers are arcs normal to the boundaries and spokes.

Figure 22. In Euclidean Möbius bands, the length of a fixed-distance curves and spokes are constant, so one type of curve is always better than the Möbius band constructed from a $2 w$ by $w$ Euclidean rectangle, in which case they are always equal. However, on that particular Möbius band, semi-circles beat out both spokes and fixed-distance curves over the entire range of areas.

One of the great things about bands is that we can use them to find the isoperimetric regions on strips, tori, cylinders, Klein bottles, and twisted chimney spaces without assuming existence and regularity. The following arguments are a generalization of Lee's proof of the isoperimetric problem in the Euclidean infinite strip [7, Proposition 4.2].

Definition 4.7. A rectangular torus is a Euclidean rectangle with opposite sides identified. A rectangular Klein bottle is Euclidean rectangle with opposite sides identified where one pair of sides is identified with a $180^{\circ}$ twist.

Definition 4.8. Consider the region of the Euclidean or hyperbolic plane between two curves of equal constant curvature. One boundary must be concave, the other convex. If we identify the boundaries, we get a cylinder. If we identify the boundaries with a $180^{\circ}$ twist with respect to some perpendicular axis, we get a two-dimensional twisted chimney space as in Figure 23.

Note that there is no such thing as a spherical cylinder or twisted chimney space, since two circles with equal curvature are both concave and hence cannot be smoothly identified.

Proposition 4.9. We can view the rectangular torus as a double of the band. If we double the isoperimetric regions of area $A$ on the band, we get isoperimetric 


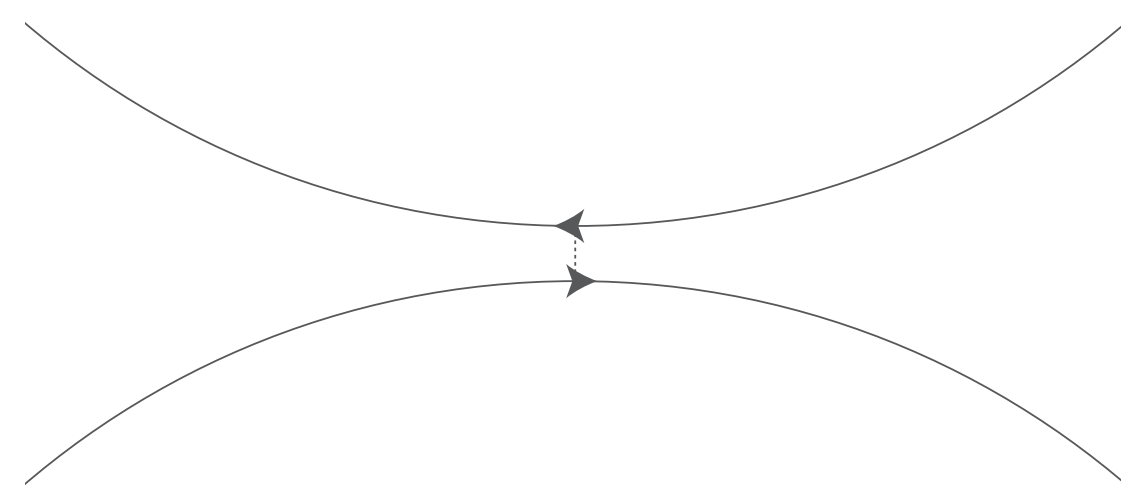

Figure 23. A two-dimensional hyperbolic twisted chimney space is made by oppositely identifying two curves of equal constant curvature.

regions of area $2 A$ on the torus, indeed all of them up to translation. Hence, the band and torus isoperimetric problems are equivalent.

Proof. Let $C$ be a minimizer in a Euclidean band enclosing area $A$. Reflect the band along one of its boundaries and identify the other two, forming a torus. $C$ and its reflection will enclose area $2 A$ in the torus.

Suppose under contradiction that the union of $C$ and its reflection is not minimizing. Then there is a set of curves $D$ enclosing area $2 A$ which is shorter than the union of $C$ and its reflection. Pick two longitudes which divide the torus into two bands of equal area. By the intermediate value theorem, we can pick our longitudes so that half of the area enclosed by $D$ lies in one band and half lies in the other. Since $D$ is shorter than twice the length of $C$, at least one of the pieces of $D$ will be shorter than $C$ but enclose the same area, contradiction. Therefore, the union of $C$ and its reflection is a minimizer in the torus.

Now let $K$ be a minimizer in a torus. Cut along two antipodal longitudes to create a pair of bands of equal area, with half of the area enclosed by $K$ in each. Suppose under contradiction that the piece of $K$ in one of the bands is not minimizing. Call that piece $K^{\prime}$.

Case 1: Suppose $K^{\prime}$ is no longer than the other piece of $K$. Since $K^{\prime}$ is not minimizing, there is a shorter set of curves $J$ enclosing the same area. Reflect the band over a boundary and identify the other two, recreating the original torus. Therefore, $J$ and its reflection are shorter than $K$ but enclose the same area, contradiction.

Case 2: Suppose $K^{\prime}$ is longer than the other piece of $K$. Take the band containing the shorter piece of $K$ and reflect it over a boundary and identify 
the other two. The shorter piece of $K$ and its reflection are shorter than $K$ but enclose the same area, contradiction.

Therefore, each piece on $K$ is a minimizer in its band.

Since every minimizer in the band can be doubled to create a minimizer in the torus and every minimizer in the torus can be cut in half to form minimizers in the band, the problems are equivalent.

Corollary 4.10. The Euclidean infinite strip and cylinder problems are equivalent. Both can be derived from extreme cases of the band.

Proof. The minimizers on a band with a long circumference will be the same as the minimizers on a strip. The minimizers on a long torus will be the same as the minimizers in a cylinder. Therefore, by Proposition 4.9 the two problems are equivalent and their solutions can be derived from the band theorem.

The band theorem also gives rise to solutions in non-orientable surfaces, given the following lemma.

Lemma 4.11. In an annulus bounded by two constant-curvature curves, a minimizer intersects a given boundary twice (if ever).

Proof. If a component intersects only one boundary, it must do so twice since it has finite length. Therefore, it is an arc normal to the boundary. Multiple arcs on a given boundary can be ruled out through translation as can an arc and a component meeting both boundaries. If a component intersects both boundaries, it is a spoke. One spoke cannot enclose any area, and three or more spokes are clearly inferior to two. Therefore, if a minimizer meets a given boundary, it must do so twice.

Proposition 4.12. We can view the rectangular Klein bottle as a double of the band. If we double the isoperimetric regions of area $A$ on the band, we get isoperimetric regions of area $2 A$ on the Klein bottle, indeed all of them up to translation. The Euclidean band and rectangular Klein bottle problems are equivalent.

Proof. Let $C$ be a minimizer in a band enclosing area $A$. Reflect the band along one of its boundaries. Together $C$ and its reflection enclose area $2 A$. Note that each boundary point of $C$ and its reflection lie on the same meridian. In order to make an opposite identification, we need to pick some meridian $M$ that will remain fixed. If $C$ does not intersect the boundary, choose any meridian to be $M$. If $C$ intersects a boundary twice, pick $M$ to be the meridian halfway between the points of intersection, so that each point will get identified to the other point's reflection. By Lemma 4.11, $M$ cannot intersect the same boundary once or more than two times. Having chosen $M$, oppositely identify two boundaries to form a Klein bottle. In this way, since every boundary point of $C$ has been identified to a boundary point in $C$ 's reflection (and vice versa), the union of $C$ and its reflection is a simple closed curve enclosing area $2 A$. The rest of the proof follows the same methods as for Proposition 4.9. 
Corollary 4.13. The Euclidean infinite strip and twisted chimney space problems are equivalent. Both can be derived from extreme cases of the band.

Proof. The minimizers on a long band with a long circumference will be the same as the minimizers on a strip. The minimizers on a long Klein bottle will be the same as the minimizers in a twisted chimney space. Therefore, by Proposition 4.9 the two problems are equivalent and their solutions can be derived from Theorem 4.2 (the band theorem).

We conclude this section with two conjectures about the nature of hyperbolic surfaces.

Conjecture 4.14. The minimizer on a hyperbolic cylinder without a cusp is a circle for small areas and two equidistant curves bounding a band for large areas. The minimizer on a hyperbolic twisted chimney space is a circle for small areas and a single equidistant curve bounding a Möbius band for large areas.

Conjecture 4.15. A hyperbolic cylinder constructed from the region between two equidistant curves is congruent to the cylinder constructed by identifying their axes. The same property holds for twisted chimney spaces.

\section{Hyperbolic genus-two surfaces}

We now consider the isoperimetric problem on the simplest hyperbolic manifold: the compact genus-two surface. All genus- $n$ surfaces have hyperbolic structures $(n>1)$ and can be decomposed into hyperbolic pairs of pants (a sphere minus three disks), as we do this section. By studying the simplest case, we hope to gain insight into how to approach the isoperimetric problem on all hyperbolic surfaces.

One common approach to studying genus- $n$ surfaces is to chop them up into simpler pieces by cutting along geodesics. Since any genus- $n$ surface can be formed from a polygon with $4 n$ sides, geometers often use the hyperbolic octagon as a road map for what is happening on the surface of the genus-two surface. We too tried that approach, but had difficulty controlling the lengths of the geodesics we were interested in. We had more success when, following William Thurston [12], we cut the surface into two pairs of pants and each pair of pants into two right-angled hexagons (Lemma 5.8). We then looked for properties of hyperbolic hexagons that we could extend to hyperbolic genus-two surfaces (Proposition 5.10).

In the following lemmas, we lay the groundwork for the isoperimetric problem with a succession of topological arguments, before moving into the geometry of curvature and length.

Lemma 5.1. Two disjoint non-separating embedded closed curves separate the genus-two surface if and only if they are in the same homotopy class. Cutting a genus-two surface along two such curves in different homotopy classes results in a four-punctured sphere. 
Proof. Two disjoint non-separating embedded closed curves in the same homotopy class clearly separate the genus-two surface. Therefore, consider two such curves in different homotopy classes. Cut along those curves. The resulting subsurfaces will have a total of four boundaries and total Euler characteristic $\chi=-2$. Thus, at least one subsurface will have $\chi \geq-2$, so it must be one of the following:

(1) disk (once-punctured sphere),

(2) annulus (twice-punctured sphere),

(3) pair of pants (thrice-punctured sphere),

(4) four-punctured sphere,

(5) once-punctured torus,

(6) twice-punctured torus.

A disk (1) requires one of the curves to be homotopically trivial and hence separating, a contradiction. An annulus (2) implies that there is a continuous deformation taking one boundary to the other which means the curves are in the same homotopy class, a contradiction. A pair of pants (3) has three boundaries, so two of the boundaries must be made from a single curve. The other curve therefore is separating, contradiction. A once-punctured torus (5) is bounded by a single curve, so that curve must be separating, a contradiction. A twice-punctured torus (6) has $\chi=-2$ so there can be no other subsurfaces, yet there are only two boundaries, a contradiction. Therefore, at least one of the subsurfaces must be four-punctured sphere (4). Since a four-punctured sphere has $\chi=-2$ it is the only subsurface. Therefore, if the curves are in different homotopy classes, they do not separate the genus-two surface, but rather split it into a four-punctured sphere.

Lemma 5.2. Three disjoint closed curves will always separate the genus-two surface.

Proof. Consider two disjoint closed curves which do not separate the surface. By Lemma 5.1 they are in different homotopy classes and cutting along them results in a four-punctured sphere. Every closed curve on a sphere is separating, regardless of whether there are punctures. Therefore, adding a third closed curve on the genus-two surface will separate it.

Lemma 5.3 ([12, Proposition 5.3.1]). On every closed hyperbolic surface, there is a unique closed geodesic in every free non-trivial homotopy class.

Lemma 5.4. On every hyperbolic genus-two surface, a separating geodesic splits the surface into two once-punctured tori, each with area $2 \pi$.

Proof. Suppose when you cut along a separating geodesic you end up with two holes on one side. The region without holes will be a disk, which has Euler characteristic $\chi=1$. However, its Gauss curvature is $G=-1$, and its boundary 
has no geodesic curvature $\left(k_{g}=0\right)$. Thus, from the Gauss Bonnet formula

$$
\int_{S} G d A=2 \pi \chi-\int_{d S} k_{g} d s
$$

we get area $A=-2 \pi$, a contradiction. Therefore, there must be a hole on each side, so a separating geodesic splits the genus-two surface into two oncepunctured tori. These two surfaces both have geodesic boundary and the same Euler characteristic, so by the Gauss-Bonnet formula they must have the same area, $2 \pi$.

The following proposition is the culmination of the preceding lemmas and provides a jumping off point for our study of hyperbolic genus-two surfaces.

Proposition 5.5. Any three disjoint closed geodesics will partition a hyperbolic genus-two surface into two pairs of pants, each with area $2 \pi$.

Proof. Case 1: Let $g_{1}$ be a separating geodesic. By Lemma 5.4, cutting along $g_{1}$ splits the surface into two once-punctured tori. Add a disjoint geodesic $g_{2}$. Since $g_{2}$ does not cross $g_{1}$ it must be entirely contained in one of the two once-punctured tori. According to the Gauss-Bonnet formula, $g_{2}$ cannot bound a disk. Since there is a unique geodesic in each homotopy class (Lemma $5.3), g_{1}$ and $g_{2}$ cannot bound an annulus. Therefore, $g_{2}$ does not separate the once-punctured torus, but rather splits it into a pair of pants. Add a third disjoint geodesic $g_{3}$. Since $g_{3}$ does not cross the other two geodesics, it must be contained in either the pair of pants or the other once-punctured torus. If we draw $g_{3}$ on the pair of pants than it will bound a disk or an annulus, a contradiction. Therefore, $g_{3}$ must go on the remaining once-punctured torus, splitting it into a second pair of pants.

Case 2: Consider three disjoint closed geodesics, none of which are separating. Pick any two of these geodesics and cut along them to get a four-punctured sphere (Lemma 5.1). By Lemma 5.2, three disjoint closed geodesics partition a genus-two surface, so the third geodesic must split the sphere into two regions. If one of the regions has no punctures it is a disk, contradiction. If it contains one puncture, it is an annulus, also a contradiction. Therefore, each region must have two punctures and by definition is a pair of pants.

Each pair of pants has geodesic boundary and the same Euler characteristic, so by the Gauss-Bonnet formula each must have the same area, $2 \pi$.

Proposition 5.5 gives us a powerful tool for solving the isoperimetric problem. Not only can we cut every genus-two surface into two pairs of pants, but we can do so using any three disjoint closed geodesics. In fact, we can find out everything we need to know about the hyperbolic structure of a given genustwo surface from the lengths of any three disjoint closed geodesics. To discover what lengths are allowed, we will cut each pair of pants into two right-angled hexagons. 
Lemma 5.6 ([4, p. 94]). A hyperbolic right-angled hexagon is determined by the lengths of three alternating sides.

Lemma 5.7. Every hyperbolic pair of pants can be cut into two identical rightangled hexagons.

Proof. A hyperbolic pair of pants is bounded by three closed geodesics. In hyperbolic surfaces, each pair of disjoint geodesics has a unique perpendicular geodesic running between them. Thus, cutting along the three perpendicular geodesics perpendicular to the boundaries will split the pair of pants into two hyperbolic right-angled hexagons. Since the two hexagons have three side lengths in common (the geodesics we cut along), by Lemma 5.6 they are identical.

The following lemma completes the connection between hyperbolic rightangled hexagons and hyperbolic genus-two surfaces.

Lemma 5.8. Every hyperbolic genus-two surface can be cut along any three disjoint closed geodesics into four right-angled hexagons. Any four identical right-angled hexagons can be identified to form a genus-two surface.

Proof. Every genus-two surface can be cut along three disjoint closed geodesics into two pairs of pants (Proposition 5.5), which in turn can be cut into four right-angled hexagons (Lemma 5.7). Likewise, two identical right-angled hexagons can be identified along three alternating sides to form a pair of pants. Doubling this pair of pants along its boundaries yields a genus-two surface.

In the next proposition, we show that the lengths of any three disjoint closed geodesics on the genus-two surface can treated as independent parameters in determining a hyperbolic structure.

Lemma 5.9 ([4, p. 99]). For every three positive numbers $a, b, c$, there is a unique convex right-angled hexagon in $\mathbb{H}^{2}$ with alternating side lengths of $a, b, c$.

Proposition 5.10. Given any three disjoint isotopy classes on the genus-two surface and any three positive numbers $a, b, c$, there exists a hyperbolic metric where the geodesics in those isotopy classes have lengths $a, b$, and $c$.

Proof. Given any three disjoint isotopy classes on the genus-two surface, we can cut along the geodesics in the three isotopy classes to get two pairs of pants (Lemma 5.8). Cut those pairs of pants to get four right-angled hexagons, whose alternating sides $s_{1}, s_{3}, s_{5}$ derive from portions of our three geodesics. Given three positive numbers $a, b, c$, by Lemma 5.9 we can scale our rightangled hexagons so that on each hexagon the sides $s_{1}, s_{3}, s_{5}$ have lengths $a, b, c$. Now re-identify the hexagons the same way we cut them to get a genustwo surface with geodesics in the same isotopy classes as before. The new genus-two surface will have geodesics in the given isotopy classes with lengths $a, b, c$, or multiples thereof. For some isotopy classes, it may be necessary to 

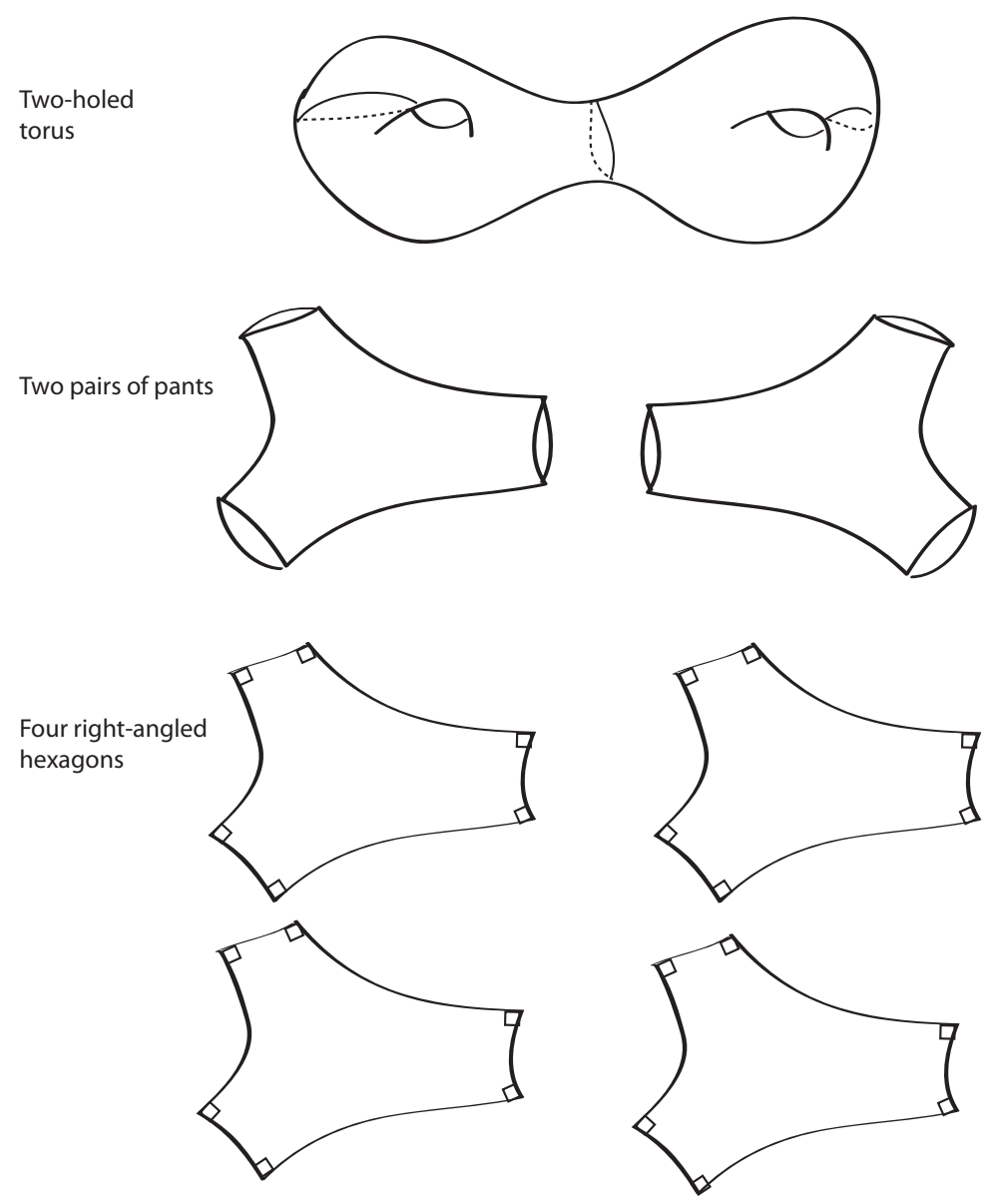

Figure 24. Every genus-two surface can be cut along three disjoint closed geodesics to form two pairs of pants, which can then be cut into four right-angled hexagons.

choose hexagon with side-lengths such as $\frac{a}{2}$ or $\frac{b}{3}$ in order to achieve the desired lengths of geodesics.

We are now ready to consider the isoperimetric problem on hyperbolic surfaces.

Theorem 5.11 ([1, Theorem 2.2]). Let $S$ be a hyperbolic surface. Given area $A<($ area $S) / 2$, a minimizer consists of precisely one of the following:

(1) a circle bounding a disk,

(2) horocycles bounding cusps,

(3) a pair of equidistant curves bounding an annulus, 
(4) lone equidistant curves.

Note: "Lone equidistant curves" means one equidistant curve per isotopy class. A pair of equidistant curves implies two curves in the same isotopy class with the same geodesic axis.

Proof sketch. Consider all hyperbolic constant-curvature curves: circles when $|\kappa|>1$, horocycles when $|\kappa|=1$, and equidistant curves when $|\kappa|<1$. Multiple circles can be ruled out through translation. Circles and horocycles cannot occur together or with equidistant curves since all three have different curvatures. Perimeter-area inequalities are then used to show that an annulus occurs alone.

Adams and Morgan also provide perimeter-area formulas for each type of minimizer.

Lemma 5.12 ([1, Lemma 2.3]). Consider a collection of equidistant curves of total length $L$, each with curvature $\kappa$ at distance $s$ from their respective geodesics. Let $l$ be the combined lengths of the underlying geodesics. Then

$$
L^{2}=\Delta A^{2}+l^{2}
$$

where $\Delta A$ is the area of the region between the curves and the geodesics. Also, $\Delta A=l \sinh s, L=l \cosh s$, and $\kappa=\tanh s$.

Now that we know what types of curves we have to work with, we can apply the results of Adams and Morgan to the genus-two surface.

Theorem 5.13. On a hyperbolic genus-two surface a minimizer of length $L$ enclosing area $A$ is one of the following:

(1) a disk with $L^{2}=A^{2}+4 \pi A$,

(2) an annulus with $L^{2}=A^{2}+4 l_{m}^{2}$ where $l_{m}$ is the length of the shortest closed geodesic,

(3) a separating curve with $L^{2}=A^{2}-4 \pi A+4 \pi^{2}+l_{s}^{2}$ where $l_{s}$ is the length of the shortest separating geodesic,

(4) a pair of pants with $L^{2}=A^{2}-4 \pi A+4 \pi^{2}+l_{t}^{2}$ where $l_{t}$ is the combined length of the three shortest non-separating geodesics.

For small areas, the minimizer is a disk. Separating curves and pairs of pants are mutually exclusive, unless $l_{s}=l_{t}$ in which case they are minimizing for the same range of areas. See Figure 1 for illustration.

Proof. Since the surface is hyperbolic, a minimizer must be one of the candidates listed in Theorem 5.11. Disks and annuli both exist on genus-two surfaces, but cusps do not. Suppose the minimizer consists of lone equidistant curves:

Case 1: If none of the curves are separating, then there must be at least three such curves to enclose area (Lemma 5.1). Since every three disjoint closed geodesics enclose a pair of pants (Lemma 5.5), every three disjoint equidistant curves do as well (4). Since there are up to three isotopy classes on the 
genus-two surface, a minimizer cannot contain more than three disjoint lone equidistant curves.

Case 2: If one of the equidistant curves is separating, then that curve splits the surface into two once-punctured tori. If there are additional equidistant curves, they must reside on different once-punctured tori since they belong to distinct isotopy classes. However, each curve will merely split its respective once-punctured torus into a pair of pants, thereby adding more perimeter without affecting the area, a contradiction. Therefore, if the minimizer contains a separating curve, then there are no other components.

Therefore, a minimizer in a hyperbolic genus-two surface is one of the types listed. The perimeter-area formulas follow immediately from Lemma 5.12.

Remark 5.14. Since disks and annuli are minimizing on the maximal genustwo surface [1, Theorem 3.1] and separating curves and pairs of pants are minimizing in certain limiting cases (Theorem 5.18), all four types of minimizers occur.

Having characterized all possible types of minimizers on hyperbolic genustwo surfaces, we will see which types occur in each of the limiting cases.

Theorem 5.15. The following are all the smooth limiting cases of hyperbolic genus-two surfaces:

(1) two once-punctured tori,

(2) a once-punctured torus and a thrice-punctured sphere,

(3) a twice-punctured torus,

(4) a 4-punctured sphere,

(5) two thrice-punctured spheres connected at one pair of cusps,

(6) two thrice-punctured spheres connected at three pairs of cusps.

Proof. We will first show that these six cases are the only ones possible. Since the hyperbolic genus-two surface has Euler characteristic $\chi=-2$, the Euler characteristic of every limiting case must also be -2 . Since there are no hyperbolic surfaces without boundary that have $\chi>0$, every component of a limiting case must have $\chi=-1$ or -2 . The only choices therefore are once-punctured tori, twice-punctured tori, thrice-punctured spheres, and fourpunctured spheres. Since none of these have $\chi>0$, a limiting case cannot include pieces with $x<-2$. Four-punctured spheres and twice-punctured tori are both limiting case candidates, since they each have $\chi=-2$, yielding cases (3) and (4). Once-punctured tori and thrice-puncture spheres have Euler characteristic -1 so they can either be combined with themselves yielding cases (1) and $(5 / 6)$ or combined with each other yielding case $(2)$.

If a limiting case has more than two cusps, we can identify them in different ways, potentially leading to more limiting cases. In case (1) with two oncepunctured tori there are only two cusps so we simply identify them to each other. In case (2) with a once-punctured torus and a thrice-punctured sphere, 


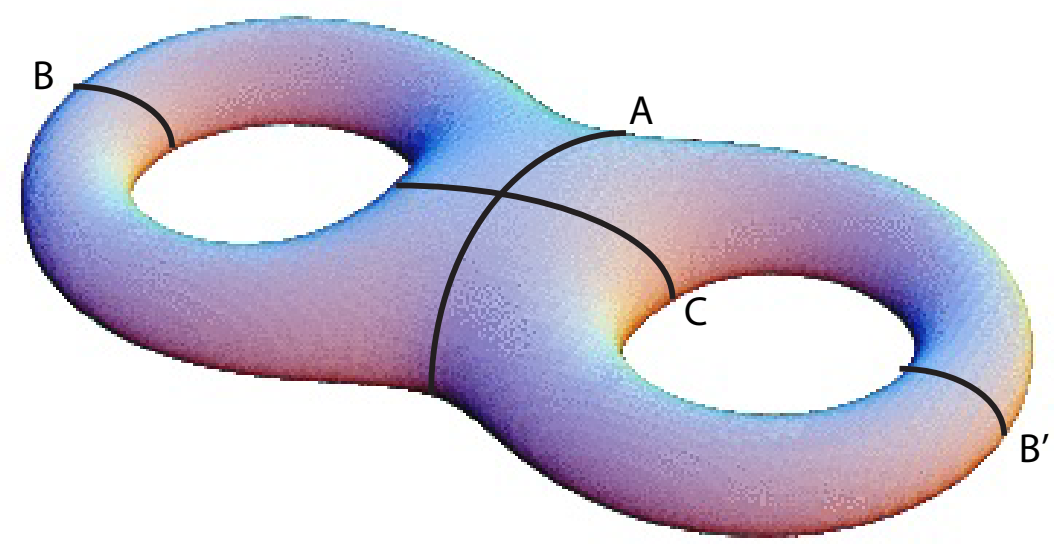

Figure 25. $A, B, B^{\prime}$, and $C$ are geodesics. Shrinking them in different combinations yields the limiting cases listed. Original picture from [13] (geodesics added).

it does not matter which of the thrice-punctured sphere's three cusps we identify to the torus's cusp, since there is an isometry of hyperbolic space taking one cusp to another. In case (3), a twice-punctured torus, there are only two cusps. In case (4), a four-punctured sphere, there are three ways to identify the cusps. All lead to different genus-two surfaces, but there does not seem to be a way to classify them in a way that will be helpful in determining their minimizers. If we have two thrice-punctured spheres, we can take one sphere and identify two of its cusps to each other and one to the other sphere, leading to case (5), or identify all three cusps with the other sphere, yielding case (6). We have now considered all possible smooth surfaces and all the ways of identifying them. Therefore, our list is complete.

We will now show that all these cases do in fact occur. From Lemma 5.10, we know that by cutting the surface into hexagons, scaling the hexagons, and regluing them, that we can make up to three disjoint closed geodesics arbitrarily short. Therefore, choose a separating geodesic (call it $A$ ) and two other disjoint closed geodesics (call them $B$ and $B^{\prime}$ ) as shown in Figure 25. If we alter the metric so that $A$ shrinks to a point we get two once-punctured tori (1). Shrinking $A$ and $B$ yields a once-punctured torus and a thrice-punctured sphere (2). Shrinking $B$ yields a twice-punctured torus (3). Shrinking both $B$ and $B^{\prime}$ yields a four-punctured sphere (4). Shrinking along $A, B$, and $B^{\prime}$ yields two thrice-punctured spheres (5). To get case (6), replace the separating geodesic with another closed geodesic with does not intersect the others $(C$ in Figure 25), and shrink all three geodesics. 


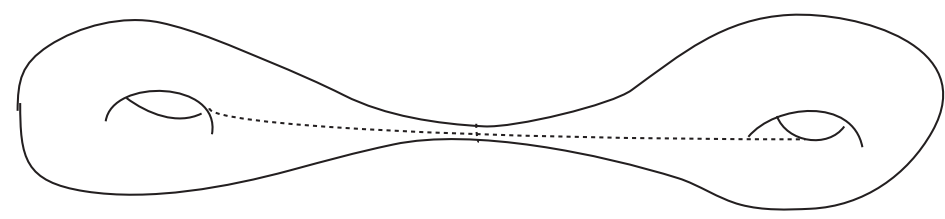

Figure 26. The collar lemma states that if a closed geodesic is very short, every closed geodesic crossing it must be very long.

Before we can determine what types of minimizers occur in each of these limiting cases, we need the following important result about disjoint closed geodesics.

Lemma 5.16. If $g$ is a simple closed geodesic shorter than 1.31, then every closed geodesic which crosses $g$ must be longer than 1.31, as in Figure 26.

Proof. According to the Collar Lemma [8, Lemma 3.2], a simple closed geodesic $g$ of length $l$ has two identical equidistant curves on each side bounding an annulus of area $l / \sinh (l / 2)$. Let $2 d$ be the width of the annulus. From Lemma 5.12 we know that the area between one of the equidistant curves and its axis is $l \sinh (d)$. Therefore, $l / \sinh (l / 2)=2 l \sinh (d)$, so

$$
d=\sinh ^{-1} \frac{1}{2 \sinh (l / 2)} .
$$

Any closed geodesic that crosses $g$ will cross the entire annulus, so its length will exceed $2 d$. Therefore, $g$ is shorter than $2 d$, every closed geodesic it crosses will have length greater than $l$. Plugging $l=2 d$ into the above equation yields $l=1.31 \cdots$. Therefore, if $g$ is shorter than 1.31 , any closed geodesic crossing $g$ must be longer than 1.31 .

Lemma 5.17. Given three non-separating disjoint closed geodesics on a genustwo surface, every other closed geodesic must cross at least one of these geodesics.

Proof. Pick three disjoint closed geodesics. Every three disjoint closed geodesics partition the genus-two surface into two pairs of pants 5.5. A pair of pants is a thrice-punctured sphere. Every closed curve on a thrice-punctured sphere will be isotopic to one of the boundaries. There is only one geodesic in each isotopy class (Theorem 5.3). Therefore, neither pair of pants can contain a closed geodesic, so every additional closed geodesic must intersect one of the geodesics we have already chosen.

Proposition 5.18. In genus-two surfaces near the limiting cases listed in Theorem 5.15, the minimizer is a disk for very small areas and an annulus for small areas. For large areas (close to $2 \pi$ ), the minimizer is 
(1) a separating curve on genus-two surfaces approaching cases (1), (2), and (5),

(2) a pair of pants on genus-two surfaces approaching case (6).

In surfaces approaching cases (3) and (4), the minimizer for large areas depends on the metric.

Proof. From Theorem 5.13 we know that the minimizer will always be a disk for very small areas. Furthermore, in each limiting case the length of the shortest geodesic $\left(l_{m}\right)$ is almost zero, so from our formulas we see that annuli are superior to disks when $A \geq l_{m}^{2} / \pi$.

In cases (1) and (2) and (5), the separating geodesic is the shortest (or equally shortest) geodesic, so clearly a separating curve is better than an annulus or disk for large areas. Suppose under contradiction that a pair of pants is also minimizing. Then by Theorem 5.13 there must be three non-separating disjoint geodesics whose combined length is shorter than the $l_{s}$. By Lemma 5.17 one of these geodesics must intersect the separating geodesic of length $l_{s}$ and by Lemma 5.16 it must be longer than $l_{s}$, contradiction. Therefore, the minimizer must be a separating curve for large areas.

If we take the separating curve and annulus equations from Theorem 5.13 and set them equal we get $A^{2}-4 \pi A+4 \pi^{2}+l_{s}^{2}=A^{2}+4 l_{m}^{2}$. In this case $l_{s}=l_{m} \approx 0$, so we end up with $A \approx \pi$. Note than $A$ never reaches $\pi$, since that would require the separating curve to have 0 length. Therefore, the minimizer is a separating curve for $A>\pi$.

Case (6) is formed by making three non-separating disjoint closed geodesics arbitrarily short (see proof of Theorem 5.15). By Lemma 5.17 every separating geodesic must cross one of these geodesics and by Lemma 5.16 must be longer than 1.31. Therefore, $l_{s}>l_{t}$ so a separating curve cannot be minimizing. From the above calculations, we see that a pair of pants is best for $A>\pi$.

Remark 5.19. Proposition 5.18 can also be used to find the minimizers on each of the punctured hyperbolic surfaces mentioned in the limiting cases. For instance, on a once-punctured torus, a minimizer can be a) horocycles bounding cusps, b) a separating curve, or c) two equidistant curves bounding a punctured annulus.

It is interesting to note that in every example we have examined, both disks and annuli are isoperimetric for some range of areas. Is this always the case? We begin our investigation with a lemma about the lengths of closed geodesics.

Lemma 5.20 ([10, Theorems 5.2, 5.3]). For a hyperbolic genus-two surface, the length of the shortest closed geodesic $l_{m}<3.058$.

Thus, no matter what our hyperbolic metric is, there will always be some closed geodesic shorter than 3.058. The result of this restriction is that disks are never optimal over the entire range of areas. 
Proposition 5.21. On every hyperbolic genus-two surface, there are at least two types of isoperimetric regions, at least one of which is a disk.

Proof. We know that disks are always minimizing for small areas (Theorem 5.13 ), and from the perimeter-area formulas, we also see that an annulus beats a disk when $A>l_{m}^{2} / \pi$. Since on every genus-two surface $l_{m}<3.058$ (Lemma $5.20)$, and $(3.058)^{2} / \pi<2 \pi$, annuli are always superior to disks for half the area of the surface. Therefore, the minimizer for half the area is something other than a disk.

By now, we know that every hyperbolic genus-two surface has at least two types of isoperimetric regions, one of which is a disk. What about the other types? Is it possible to have a hyperbolic genus-two surface with no isoperimetric annuli?

Lemma 5.22. A genus-two surface has no isoperimetric annuli if and only if

(1) $2.37<l_{s}<5.94$,

(2) $2.37<l_{m}$, and

(3) $l_{s}^{2} \leq 8 l_{m}^{2}-4 \pi^{2}$.

Furthermore, there are no genus-two surfaces for which only disks and pairs of pants are isoperimetric.

Proof. Case 1: Consider all genus-two surfaces for which separating curves are superior to pairs of pants. Then an annulus is never isoperimetric if and only if it is inferior to both disks and separating curves for every area. From Theorem 5.13 we see that annuli lose to disks if and only if $A \leq l_{m}^{2} / \pi$ and that annuli lose to separating curves if and only if $A \geq\left(4 l_{m}^{2}-4 \pi^{2}-l_{s}^{2}\right) /(-4 \pi)$. Therefore, on a given genus-two surface, annuli never are isoperimetric if and only if

$$
\frac{4 l_{m}^{2}-4 \pi^{2}-l_{s}^{2}}{-4 \pi} \leq A \leq \frac{l_{m}^{2}}{\pi}
$$

which is equivalent to

$$
l_{s}^{2} \leq 8 l_{m}^{2}-4 \pi^{2}
$$

By definition, a separating curve cannot be shorter than the shortest closed geodesic, $l_{m}$. We also know that from Lemma 5.20 that $l_{m}<3.058$. Therefore, on a given genus-two surface, annuli will never be minimizing if and only if

$$
l_{m}^{2} \leq l_{s}^{2} \leq 8 l_{m}^{2}-4 \pi^{2} \leq 35.28
$$

The three stated conditions follow immediately.

Case 2: Consider all genus-two surfaces for which separating curves are not superior to pairs of pants. Then all the inequalities above are the same except we replace $l_{s}$ with $l_{t}$, the combined length of the three shortest nonseparating geodesics that enclose a pair of pants. Naturally, $l_{t}$ must be at least three times the length of the shortest closed geodesic, $l_{m}$. Therefore, $\left(3 l_{m}\right)^{2} \leq l_{s}^{2} \leq 8 l_{m}^{2}-4 \pi^{2}$, which results in $l_{m}^{2} \leq-4 \pi^{2}$, a contradiction. Thus, if separating curves are not superior to pairs of pants then isoperimetric annuli 

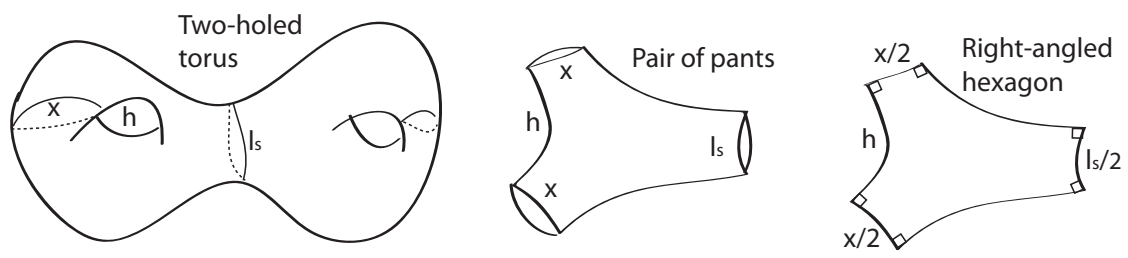

Figure 27. Cut along the shortest separating geodesic and two other geodesics to get a pairs of pants, and cut the pants to get a hexagon. Label the sides as shown. Note the obvious correspondence between the $l_{s}, l_{m}$, and the side-lengths.

occur. Therefore, there are no genus-two surfaces with only pairs of pants and disks as minimizers.

Theorem 5.23. For every hyperbolic metric on a genus-two surface, there exists a range of areas for which annuli are isoperimetric.

Proof. Suppose there exists a genus-two surface $S$ with no isoperimetric annuli. Cut $S$ along its shortest separating geodesic (length $l_{s}$ ) to obtain two oncepunctured tori. Cut one of the tori along a closed geodesic to obtain two pairs of pants. On each pair of pants, two boundaries will have the same length since they were cut from the same geodesic; the other boundary will have length $l_{s}$ as in Figure 27. Cut one of the pairs of pants into two identical right-angled hexagons. Each hexagon will have two alternating sides of the same length (which we call $x$ ) and a third alternating side of length $l_{s} / 2$. Note that the first two sides are formed from half of a closed geodesic in $S$, so $x \geq l_{m} / 2$ (where $l_{m}$ is the length of the shortest closed geodesic). The side opposite the third side is formed from an entire closed geodesic in $S$, so its length $h \geq l_{m}$.

Given $l_{s}$ and $x$, we can find $h$ using hyperbolic trigonometry [4, p. 99]:

$$
h\left(x, l_{s}\right)=\operatorname{arccosh}\left(\frac{\cosh \left(l_{s} / 2\right)+\cosh ^{2}(x)}{\sinh ^{2}(x)}\right)
$$

By Lemma 5.22(1), 2.37 $<l_{s}<5.94$. By condition $(2), l_{m}>2.37$, so $x \geq l_{m} / 2 \geq 1.18$. Suppose $x=1.18$. Then from Figure 28 we see that whenever $l_{s}<5.60$, then $l_{m} \leq h<2.37$, contradiction. Suppose $x \geq 1.18$. From Figure 29 we see that making $x$ bigger simply makes $h$ even smaller, so condition (2) still fails. Therefore, in order to satisfy condition (2), we need $5.60 \leq l_{s}<5.94$.

If $5.60 \leq l_{s}<5.94$, then by condition (3) $(5.60)^{2} \leq l_{s}^{2} \leq 8 l_{m}^{2}-4 \pi^{2}$. Hence $l_{m} \geq 2.98$. Note that $x \geq l_{m} / 2 \geq 1$.49. In Figure 30 , we see that if $x=1.49$, $h<2.98$ and hence $h<l_{m}$, contradiction. If $x>1.49$, then $h$ is even smaller, so the contradiction still holds.

Therefore, there is no genus-two surface that satisfies all three conditions of Lemma 5.22, so every genus-two surface must have isoperimetric annuli. 


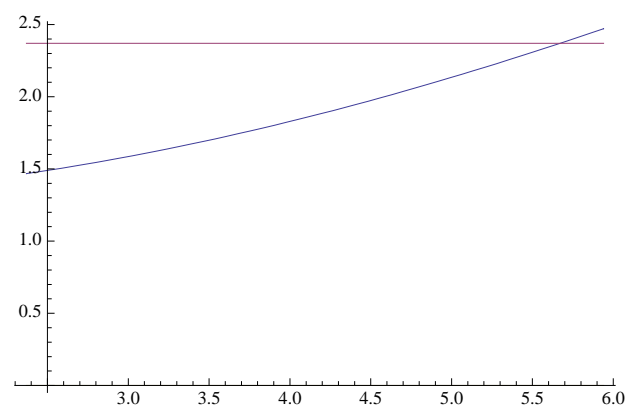

FigURE 28. $h\left(1.18, l_{s}\right)$

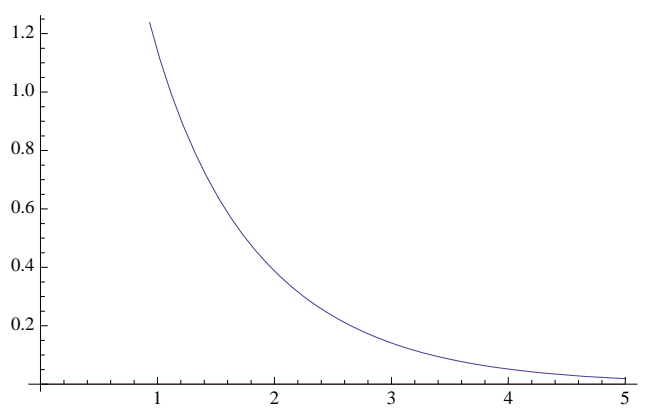

Figure 29. $h(x, 0)$. In the first graph, we pick a value for $l_{s}$ (in this case, 0 ) and graph $h$ as a function of $x$. In fact, no matter what value we pick for $l_{s}$, the graph will be strictly decreasing over the entire domain of $x$.

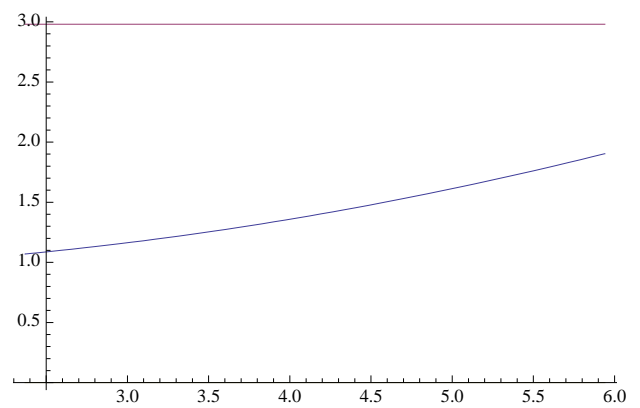

FiguRE $30 . h\left(1.49, l_{s}\right)$

We summarize the preceding three results in the following theorem. 
Theorem 5.24. On a hyperbolic genus-two surface the set of minimizers is one of the following:

(1) disks and annuli,

(2) disks, annuli, and separating curves,

(3) disks, annuli, and pairs of pants,

(4) disks, annuli, separating curves, and pants.

At least (1)-(3) occur. In case (4), separating curves and pairs of pants are minimizing for the same range of areas.

Proof. From Theorem 5.13 we know that disks are always minimizing for small areas. From Lemma 5.21 we know that disks are never minimizing for half the area of the surface, so there must always be at least two types of minimizers. Theorem 5.23 tells us that annuli must always be minimizing. Therefore, the combinations listed are the only ones possible. Disks and annuli (1) are minimizing on the maximal genus-two surface [1, Theorem 3.1], while (2) and (3) occur in the limiting cases (Proposition 5.18). Case (4) requires that the length of the shortest separating geodesic, $l_{s}$, equal the lengths of the three shortest non-separating geodesics, $l_{t}$, and that annuli are not minimizing for $A=2 \pi$ (we have yet to find a genus-two surface where this is the case).

We conclude with a peek into how the study of genus-two surfaces might be generalized to other genuses.

Proposition 5.25. On every rectangular torus, both disks and annuli occur as isoperimetric regions.

Proof. It is well known that the only minimizers on a rectangular torus are disks and annuli. As usual, disks will always occur for small areas. Therefore, all we need to show is that annuli are better than disks for some ranges of areas on every torus. Let $T$ be a torus constructed from a rectangle of length $a$ and width $b$, where $a \leq b$. Therefore, for a disk to be minimizing, its perimeter must be shorter than $2 a$, the length of an annulus. Since the area of the torus is $a b$, a disk enclosing half the area must have length $L=\sqrt{4 \pi(a b / 2)}=\sqrt{2 \pi a b} \geq$ $\sqrt{2 \pi a^{2}}>\sqrt{4 a^{2}}=2 a$. Thus, a disk will never be minimizing for half the area, so there will always be a range of areas for which annuli are minimizing.

Conjecture 5.26. For every hyperbolic metric on a genus-n surface, both disks and annuli are isoperimetric.

To readers seeking to prove or refine the above conjecture, we wish you the best of luck in your endeavors.

Acknowledgements. I would like to thank Frank Morgan, Colin Adams, Mihai Stoiciu, Shawn Rafalsky, Conor Quinn, and Colin Carroll for their help and encouragement. 


\section{References}

[1] C. Adams and F. Morgan, Isoperimetric curves on hyperbolic surfaces, Proc. Amer. Math. Soc. 127 (1999), no. 5, 1347-1356.

[2] S. S. Chern, Studies in Global Geometry and Analysis, Englewood Clifts, NJ: Math. Assoc. Am., 1967.

[3] M. Engelstein, A. Marcuccio, Q. Maurmann, and T. Pritchard, Isoperimetric problems on the sphere and on surfaces with density, New York J. Math. 15 (2009), 97-123.

[4] W. Fenchel, Elementary Geometry in Hyperbolic Space, Walter de Gruyter, 1989.

[5] J. Hass and F. Morgan, Geodesics and soap bubbles in surfaces, Math. Z. 223 (1996), no. $2,185-196$.

[6] H. Howards, M. Hutchings, and F. Morgan, The isoperimetric problem on surfaces, Amer. Math. Monthly 106 (1999), no. 5, 430-439.

[7] M. Lee, Isoperimetric regions in surfaces and in surfaces with density, Rose-Hulman Und. Math. J. 7 (2006), no. 2, www.rose-hulman.edu/mathjournal/v7n2.php.

[8] G. Mondello, A criterion for convergence in the augmented Teichmüller Space, Bull. Lond. Math. Soc. 41 (2009), 733-746.

[9] F. Morgan, Geometric Measure Theory, A beginner's guide. Fourth edition. Elsevier/ Academic Press, Amsterdam, 2009.

[10] P. Schmutz, Riemann surfaces with shortest geodesic of maximal length, Geom. Funct. Anal. 3 (1993), no. 6, 564-631.

[11] M. F. da Silva, Isoperimetric regions in $H^{2}$ between parallel horocycles, ArXiv.org (2009), based on doctoral thesis (2006).

[12] W. P. Thurston, The Geometry and Topology of Three-Manifolds, electronic version 1.1, March 2002, http://www.msri.org/publications/books/gt3m/.

[13] E. W. Weisstein, Double Torus, From MathWorld-A Wolfram Web Resource. http:// mathworld.wolfram.com/DoubleTorus.html.

[14] J. Wiegert, The sagacity of circles: a history of the isoperimetric problem, Convergence (2004), Math. Assoc. Amer. http://mathdl.maa.org/mathDL/46/?pa=content\&sa= viewDocument\&nodeId=2344.

Department of Mathematics

Milton ACADEMY

Milton, MA 02186, USA

E-mail address: matthew_simonson@milton.edu 